\title{
Agglomeration patterns in a multi-regional economy without income effects*
}

\author{
José Gaspar; Sofia B. S. D. Castro
}

15th June 2017

\begin{abstract}
We study the long-run spatial distribution of industry using a multi-region coreperiphery model with quasi-linear log utility (Pflüger, 2004). We show that a distribution in which industry is evenly dispersed among some of the regions while the other regions have no industry cannot be stable. A spatial distribution where industry is evenly distributed among all regions except one can be stable, but only if that region is significantly more industrialized than the other regions. When trade costs decrease, the type of transition from dispersion to agglomeration depends on the fraction of workers that are mobile. If this fraction is low, the transition from dispersion to agglomeration is catastrophic once dispersion becomes unstable. If it is high, there is a discontinuous jump to partial agglomeration in one region and then a smooth transition until full agglomeration. Finally, we find that mobile workers benefit from more agglomerated spatial distributions whereas immobile workers prefer more dispersed distributions. The economy as a whole shows a tendency towards over-agglomeration for intermediate levels of trade costs.
\end{abstract}

Keywords: Core-periphery model, Footloose Entrepreneur, Multiple regions.

JEL Classification Numbers: R10, R12, R23.

${ }^{*}$ We are grateful to Pascal Mossay, Sergey Kokovin, and two anonymous referees for very useful comments and suggestions. We also thank the audience at the 2014 "Industrial Organization and Spatial Economics" conference in Saint Petersburg. This research was financed by Portuguese Public Funds through Fundação para a Ciência e Tecnologia in the framework of projects PEst-OE/EGE/UI4105/2014 and PEst-C/MAT/UI0144/2011, and Ph.D. scholarship SFRH/BD/90953/2012. Part of this work was developed while João Correia-da-Silva was a Marie Curie Fellow at Toulouse School of Economics, financed by the European Commission (H2020-MSCA-IF-2014-657283).

${ }^{\dagger}$ CEF.UP and Faculty of Economics, University of Porto. Católica Porto Business School, Universidade Católica Portuguesa. Email: jgaspar.phd@fep.up.pt

${ }^{\ddagger}$ CMUP and Faculty of Economics, University of Porto. Email: sdcastro@fep.up.pt

${ }^{\S}$ CEF.UP and Faculty of Economics, University of Porto. Email: joao@fep.up.pt 


\section{Introduction}

New Economic Geography (NEG) has been on the forefront in recent decades as an economics subject that seeks to explain the spatial distribution of economic activity. Many theoretical models have been built along the lines of the seminal Core-Periphery (CP) model proposed by Krugman (1991), in which skilled labour mobility combined with a general equilibrium framework under increasing returns, monopolistic competition and transport costs contribute to explain how demand linkages and supply linkages interplay to determine the geographical distribution of industry (Fujita et al., 1999; Ottaviano et al., 2002; Forslid and Ottaviano, 2003; Pflüger, 2004).

Most of these models predict a catastrophic transition from an evenly dispersed distribution into agglomeration in a single region as trade costs fall below a critical level. One exception is the Footloose Entrepreneur (FE) model with quasi-linear log utility $(\mathrm{QL}$ model) proposed by Pflüger (2004), where the absence of income effects on regional demand for manufactures, due to the assumption of a quasi-linear upper-tier utility function, significantly simplifies the analysis. This model reverses the predictions of the seminal CP model (Krugman, 1991) and of the original FE model (Forslid and Ottaviano, 2003), according to which industry is either evenly dispersed between the two regions or fully agglomerated in one region, and the transition between these two extreme distributions is catastrophic. In the QL model, partial agglomeration can be stable, and agglomeration is a smooth and gradual process. ${ }^{1}$

Although insightful, the lack of a multi-regional framework in the QL model potentially overlooks complex interdependencies among different regions, which do not arise in the 2-region set-up (Fujita et al., 1999; Fujita and Mori, 2005; Tabuchi et al., 2005; Behrens and Thisse, 2007; Fujita and Thisse, 2009; Behrens and Robert-Nicoud, 2011; Tabuchi, 2014). Moreover, the consideration of only two regions hinders empirical work because the real world is heterogeneous and multi-regional (Bosker et al., 2010).

This motivates us to extend the QL model (Pflüger, 2004) to any finite number of equidistant regions. The assumption of equidistant regions coupled with the removal of income effects on the demand for manufactures allows us to obtain explicit expressions for the indirect utilities of inter-regionally mobile workers and to fully characterize the stability of several kinds of spatial equilibria (one of which is novel) under general parameter values. $^{2}$ Moreover, we are able to study how the type of transition from dispersion to

\footnotetext{
${ }^{1}$ The influence of preferences on the long-run distribution of industry and on the type of transition dynamics was widely discussed by Pflüger and Südekum (2008).

${ }^{2}$ This is an important departure from Tabuchi's (2014) multi-regional analysis, which focuses on limit cases for the trade costs.
} 
agglomeration, as trade costs decrease, depends on the global size of the inter-regionally immobile (unskilled) workforce relative to the mobile (skilled) workforce.

Castro et al. (2012) and Gaspar et al. (2013) provided numerical evidence that, in the 3-region CP model and the 3-region FE model, a region without industry and two evenly populated regions cannot be a stable outcome. We provide an analytical confirmation of this result in the $n$-region QL model: at least one empty region paired with a symmetric distribution of industry among the other regions cannot be a stable outcome.

A feature of the QL model is that interior asymmetric distributions of industry may be stable. For simplicity, we focus on the one-dimensional subspace of $n-1$ evenly populated regions and one possibly asymmetric region. ${ }^{3}$ In this subspace, entrepreneurs face two decisions: that of migrating between evenly populated regions; and that of migrating between the asymmetric region and any of the evenly populated regions. ${ }^{4}$ For some parameter values, there is a stable equilibrium in which industry is partially agglomerated in a single region and evenly dispersed among the other regions. By contrast, an equilibrium where the asymmetric region has less industry than each of the other regions cannot be stable.

We show that the QL model with $n \geq 3$ exhibits a primary transcritical bifurcation at the symmetric equilibrium and a secondary saddle-node bifurcation at an interior asymmetric equilibrium, a feature which suggests that the migration dynamics of entrepreneurs as trade costs decrease are complex. The saddle-node bifurcation implies that industry will stay fully dispersed even after trade costs have fallen below the threshold level that deems a partial agglomeration equilibrium stable. However, if the industry is initially at a partial agglomeration equilibrium, a temporary rise in trade costs will make industry permanently disperse across regions.

When trade costs decrease, the transition from dispersion to agglomeration depends on the global size of inter-regionally immobile (unskilled) labour relative to mobile (skilled) labour. If it is relatively high (low worker mobility), there is catastrophic agglomeration in a single region once dispersion loses stability. If it is relatively low (high worker mobility), there is a discontinuous jump from dispersion to partial agglomeration, and a smooth transition towards agglomeration thereafter. Finally, for even lower ratios between immobile and mobile labour, dispersion is not possible, and the only possible change as trade costs fall is a smooth transition from partial agglomeration towards full agglomeration.

\footnotetext{
${ }^{3}$ In an equidistant $n$-region model, there are many other invariant subspaces. For example, any subspace where $k$ regions have the same size and the other $n-k$ regions are also equally sized. We focus on the particular case where $k=1$.

${ }^{4}$ This is true for any $n \geq 3$ because the dimension of this particular subspace is invariant in the number of regions. In a 2-region model, there is a single decision.
} 
We find that full dispersion yields the worst possible welfare to entrepreneurs. Still, even if migration increases their average utility, it will not occur if the utility of the migrant decreases (in that case, dispersion is stable). For unskilled workers it is the opposite: they attain their highest welfare at full dispersion. For the population as a whole, we show that agglomeration (partial or full), even when stable, may be socially inferior to more symmetric distributions. We conclude that the multi-regional QL model exhibits a tendency towards over-agglomeration for intermediate levels of transport costs.

Extensions of NEG models to multi-regional settings have been made, each with its own specificities. ${ }^{5}$ Some considered a "racetrack economy", that is, regions equally spaced around a circumference (Krugman, 1993; Fujita et al., 1999; Picard and Tabuchi, 2010; Castro et al., 2012; Mossay, 2013). In particular, Castro et al. (2012) studied a version of Krugman's CP model with 3 or more regions and concluded that additional regions favour agglomeration and discourage dispersion of economic activity. Akamatsu et al. (2012) and Ikeda et al. (2012) showed that in a $2^{n}$-region CP model decreasing trade costs leads to spatial period doubling agglomeration, whereby after each bifurcation the number of regions where firms locate is reduced by half and the spacing between populated regions doubles. Heterogeneity in location has also been tackled, in several ways, by considering: different network topologies to explain locational advantages of some regions (Barbero and Zofío, 2012), equally spaced regions along a line segment (Ago et al., 2006), or hexagonal configurations in a triangular space (Ikeda et al., 2014). Along different lines, Oyama (2009) considered an equidistant multi-regional CP model with self-fulfilling expectations in migration that lead to global stability of a single core region. Tabuchi and Thisse (2011) have built an NEG model that accounts for the rise of a hierarchical system of central places in a multi-regional set-up. Recently, Tabuchi (2014) used a multi-regional version of Krugman's (1991) CP model to show that it can account for the historical trend of agglomeration in the capital regions over the past few centuries. ${ }^{6}$

Tabuchi et al. (2005) also developed a multi-region model with equidistant regions and quasi-linear utility, but considered quadratic sub-utility, as in Ottaviano et al. (2002), instead of the logarithm of a CES aggregator, as in Pflüger (2004). They also considered urban congestion costs (housing and commuting), which act as an additional dispersion force. Studying the impact of falling transport costs on the size and number of cities

\footnotetext{
${ }^{5}$ However, the main conclusions of 2-region NEG models have not been reversed by the consideration of multiple regions (Bosker et al., 2010). This includes not only the equidistant $n$-region model by Puga (1999), but also models with non-equidistant regions.

${ }^{6}$ Several other works considering multi-regional NEG models could be worth mentioning (e.g., Behrens et al., 2006; Forslid and Okubo, 2012; Fabinger, 2015; Commendatore et al., 2015a). For an extensive review of the literature concerning multi-regional models, see Commendatore et al. (2015b).
} 
(non-empty regions), Tabuchi et al. (2005) found that cities initially grow in size and then shrink at a later stage, a situation which corresponds to agglomeration followed by re-dispersion of industry. Their results are driven by the interplay between inter-regional transport costs and urban congestion costs. In contrast, our results do not hinge on the existence of urban congestion costs.

The rest of the paper is organized as follows. Section 2 presents the $n$-region FE model with quasi-linear log utility and characterizes the short-run equilibrium of the model. Section 3 analyzes the stability of several types of long-run equilibria: agglomeration, dispersion, boundary dispersion, and partial agglomeration. Bifurcation patterns are described and explained in section 4 . Section 5 is dedicated to welfare analysis, first distinguishing between mobile and immobile workers, and then considering the economy as a whole. Section 6 contains some concluding remarks.

\section{The Quasi-linear Log model with $n$ regions}

The model is a natural extension of the FE model with QL utility (Pflüger, 2004) to an economy with a finite number of equidistant regions, $N=\{1, \ldots, n\}$. We omit calculations that are well known whenever reasonable. There is a mass $H$ of entrepreneurs, who can move freely among regions $\left(H=H_{1}+H_{2}+\ldots+H_{n}\right)$; and a mass $L$ of unskilled workers, who are immobile and evenly distributed across all regions $\left(L_{i}=L / n, \forall i \in N\right)$.

\subsection{Demand and indirect utility}

The preferences of all agents are represented by a quasi-linear upper-tier utility function with logarithmic sub-utility, as in Pflüger (2004):

$$
U=\mu \ln M+A, \quad 0<\mu<1
$$

where $A$ is the consumption of agricultural products and $M$ is the consumption of a CES composite of differentiated varieties of manufactures, defined by:

$$
M=\left[\int_{s \in S} d(s)^{\frac{\sigma-1}{\sigma}} d s\right]^{\frac{\sigma}{\sigma-1}}
$$

where $d(s)$ is the consumption of variety $s \in S, S$ is the mass of existing varieties, and $\sigma>1$ is the constant elasticity of substitution between varieties.

Let $p_{i}(s)$ and $d_{i}(s)$ denote delivered price and demand of variety $s$ in region $i$. The 
regional price index associated with the composite good (2) in region $i \in N$ is:

$$
P_{i}=\left[\int_{s \epsilon S} p_{i}(s)^{1-\sigma} d s\right]^{\frac{1}{1-\sigma}}
$$

A consumer in region $i \in N$ maximizes utility subject to the budget constraint:

$$
P_{i} M+A=y
$$

where $y$ is her income, $P_{i}$ is given by (3), and the price of the agricultural good is, as usual, normalized to unity. This yields the following demand:

$$
d_{i}(s)=\mu \frac{p_{i}(s)^{-\sigma}}{P_{i}^{1-\sigma}}, \quad M=\mu P_{i}^{-1}, \quad A=y-\mu .
$$

Notice that individual consumption of the agricultural good is non-negative if and only if nominal wages (income) of entrepreneurs and unskilled workers, $w_{i}$ and $w_{i}^{L}$, respectively, are not lower than $\mu$. In Section 2.2 we check that $w_{i}^{L}=1>\mu$, and in Section 2.3 we make an assumption which implies that $w_{i} \geq \mu$.

From (1) and (4), we obtain the indirect utility in region $i$ :

$$
V_{i}=y-\mu \ln P_{i}+\mu(\ln \mu-1) .
$$

\subsection{Supply}

The agricultural good is produced using one unskilled worker (farmer) for each unit that is produced (constant returns to scale), and is freely traded across the $n$ regions. Absence of trade costs implies that the price of the agricultural good is the same everywhere $\left(p_{1}^{A}=p_{2}^{A}=\ldots=p_{n}^{A}\right)$, which justifies choosing it as numeraire. Marginal cost pricing implies that the nominal wage of unskilled workers is the same in all regions and equal to the price of the agricultural good: $w_{i}^{L}=p_{i}^{A}=1, \forall i \in N$.

Note that we are assuming that the agricultural good is produced in all regions. This is true if global consumption of agricultural goods exceeds total production in $n-1$ regions. Given individual demand in (4), global consumption of agricultural goods is $\bar{w} H+L-(H+L) \mu$, where $\bar{w} \equiv \frac{1}{H} \sum_{i=1}^{n} H_{i} w_{i}$ is the weighted average nominal wage of entrepreneurs, while total production of agricultural goods in $n-1$ regions is at most $L(n-1) / n$. We will show (Proposition 1 ) that $\bar{w}=\frac{\mu}{\sigma}\left(1+\frac{L}{H}\right)$. The non-full-specialization 
(NFS) condition (Baldwin et al, 2004), which we assume henceforth, is then given by:

$$
\lambda>\frac{\mu \frac{\sigma-1}{\sigma}}{\frac{1}{n}-\mu \frac{\sigma-1}{\sigma}},
$$

where $\lambda=L / H$ is the global unskilled (immobile) to skilled (mobile) labour ratio.

Production of a variety of manufactures requires $\alpha$ units of skilled labour and $\beta$ units of unskilled labour for each unit that is produced (Forslid and Ottaviano, 2003). Therefore, the production cost of a firm in region $i$ is:

$$
C_{i}\left(x_{i}\right)=w_{i} \alpha+\beta x_{i}
$$

The iceberg cost parameter $\tau_{i j}$ denotes the number of units that must be produced in region $i$ for each unit that is delivered at region $j \in N$. We assume that trade costs are the same between any pair of regions. If $i=j$, then $\tau_{i j}=1$. If $i \neq j$, then $\tau_{i j}=\tau \in(1,+\infty)$.

A firm producing variety $s$ in region $i$ maximizes the following profit function:

$$
\pi_{i}(s)=\sum_{j=1}^{n} d_{j}(s)\left(H_{j}+\frac{L}{n}\right)\left[p_{j}(s)-\tau_{i j} \beta\right]-\alpha w_{i},
$$

The first order condition for maximization of (7) yields the following prices:

$$
p_{i}(s)=\beta \frac{\sigma}{\sigma-1} \text { and } p_{j}(s)=\tau \beta \frac{\sigma}{\sigma-1}, \forall j \neq i
$$

In any region $j \in N$, all varieties produced in region $i$ are sold at the same delivered price, $p_{i j}$, and have the same demand, $d_{i j}$.

Using (8), and the fact that the number of varieties manufactured in region $i$ is $S_{i}=$ $H_{i} / \alpha$, the price index of the composite good (3) becomes:

$$
P_{i}=\frac{\beta \sigma}{\sigma-1}\left(\frac{1}{\alpha} \sum_{j=1}^{n} \phi_{i j} H_{j}\right)^{\frac{1}{1-\sigma}}
$$

where $\phi_{i j} \equiv \tau_{i j}^{1-\sigma} \in(0,1]$ represents the "freeness of trade" between regions $i$ and $j$. Note that $\phi_{i j}=\phi \equiv \tau^{1-\sigma}$ whenever $i \neq j$, while $\phi_{i j}=1$ whenever $i=j$. 


\subsection{Short-run equilibrium}

Free entry in the manufacturing sector implies zero profit in equilibrium. Operating profits must thus exactly compensate fixed costs, which are the wages paid to entrepreneurs:

$$
\alpha w_{i}=\sum_{j=1}^{n} d_{i j}\left(\frac{L}{n}+H_{j}\right)\left(p_{i j}-\tau_{i j} \beta\right),
$$

which becomes, using (4) and (8):

$$
w_{i}=\frac{\mu}{\alpha \sigma} \sum_{j=1}^{n} \frac{p_{i j}^{1-\sigma}}{P_{j}^{1-\sigma}}\left(\frac{L}{n}+H_{j}\right),
$$

Replacing (8) and (9) in (10) we obtain:

$$
w_{i}=\frac{\mu}{\sigma} \sum_{j=1}^{n} \frac{\phi_{i j}\left(L / n+H_{j}\right)}{\sum_{m=1}^{n} \phi_{m j} H_{m}} .
$$

We describe the spatial distribution of industry by working with the share of entrepreneurs residing in each region $i$, denoted $h_{i} \equiv H_{i} / H$. The set of possible spatial distributions is the $(n-1)$-dimensional simplex defined by $\triangle=\left\{h \in \mathbb{R}_{+}^{n}: \sum_{i=1}^{n} h_{i}=1\right\}$.

As a function of $h$, the nominal wage in region $i$ can be expressed as:

$$
w_{i}=\frac{\mu}{\sigma} \sum_{j=1}^{n} \frac{\phi_{i j}\left(\lambda / n+h_{j}\right)}{\phi+(1-\phi) h_{j}}
$$

and the price index can be written as:

$$
P_{i}=\frac{\sigma \beta}{\sigma-1}\left(\frac{H}{\alpha}\right)^{\frac{1}{1-\sigma}}\left[\phi+(1-\phi) h_{i}\right]^{\frac{1}{1-\sigma}} .
$$

Recall that individual consumption of the agricultural good is positive if and only if the nominal wage is greater than $\mu$. This is always the case for unskilled workers, because $w_{i}^{L}=1>\mu$. Regarding entrepreneurs, $\lambda \geq \sigma / \phi-1$ is a sufficient condition for $w_{i} \geq \mu$, since, from inspection of (12), $w_{i} \geq \frac{\mu}{\sigma} \sum_{j=1}^{n} \phi_{i j}\left(\lambda / n+h_{j}\right) \geq \frac{\mu \phi}{\sigma}(\lambda+1)$.

Proposition 1. The weighted average nominal wage paid to entrepreneurs at any spatial distribution is given by:

$$
\bar{w}=\frac{\mu}{\sigma}(1+\lambda) .
$$

Proof. See Appendix A. 
The indirect utility of entrepreneurs becomes, after replacing (13) in (5):

$$
V_{i}=w_{i}+\frac{\mu}{\sigma-1} \ln \left[\phi+(1-\phi) h_{i}\right]+\eta
$$

where $\eta \equiv-\mu \ln \left(\frac{\sigma \beta}{\sigma-1}\right)+\frac{\mu}{\sigma-1} \ln \left(\frac{H}{\alpha}\right)+\mu(\ln \mu-1)$ is a constant.

Replacing the expression for the nominal wage (12), we obtain:

$$
V_{i}=\frac{\mu}{\sigma} \sum_{j=1}^{n} \frac{\phi_{i j}\left(\lambda / n+h_{j}\right)}{\phi+(1-\phi) h_{j}}+\frac{\mu}{\sigma-1} \ln \left[\phi+(1-\phi) h_{i}\right]+\eta .
$$

\section{Long-run equilibria}

The replicator dynamics are generally well suited to describe the migration of entrepreneurs when they are short-sighted (Baldwin et al., 2004). The rate of change of the share of entrepreneurs in a region $i$ is assumed to be proportional to the difference between region $i$ 's indirect utility, $V_{i}$, and the weighted average indirect utility across all regions, $\bar{V}(h)=\sum_{j=1}^{n} h_{j} V_{j}(h)$. We thus consider a dynamical system given by:

$$
\dot{h}_{i}=h_{i}\left[V_{i}(h)-\bar{V}(h)\right], \quad \forall i \in N \backslash\{n\},
$$

which, since $h_{n}=1-\sum_{i \neq n} h_{i}$, implies that $\dot{h}_{n}=-\sum_{i \neq n} \dot{h}_{i}=h_{n}\left[V_{n}(h)-\bar{V}(h)\right]$.

A spatial distribution $h \in \Delta$ is said to be an equilibrium if $\dot{h}=0$. The boundaries of $\triangle$ are invariant for the dynamics. If a region is initially empty, it will remain so unless some exogenous migration to that region occurs. ${ }^{7}$ Moreover, every spatial distribution $h \in \Delta$ such that $h_{i}=1 / k$ in $k$ regions (and $h_{i}=0$ in the other) is an equilibrium.

Our first goal is to provide a description of stable configurations of mobile workers in the economy. This is achieved by studying the local stability of several types of equilibria. First, we consider full agglomeration in a single region $i\left(h_{i}=1\right)$. Then, we study symmetric dispersion, where entrepreneurs are evenly distributed among all regions $\left(h_{i}=\right.$ $1 / n, \forall i)$. We also study boundary dispersion, where entrepreneurs are evenly dispersed among some of the regions $\left(h_{j}=1 / k\right.$ in $k \in\{2, \ldots, n-1\}$ regions and $h_{i}=0$ elsewhere). The last class of equilibria we study are asymmetric interior distributions where one region has a share of entrepreneurs $h_{i} \in(0,1)$ and the other $n-1$ regions are evenly populated with shares $\left(1-h_{i}\right) /(n-1)$. We refer to this spatial distribution as partial agglomeration.

An equilibrium is said to be stable if, after a small perturbation of the equilibrium

\footnotetext{
${ }^{7}$ This may seem unreasonable if an empty region has a positive utility differential. However, the replicator dynamics are generally used to capture the effect of migration driven by imitation, so a possible interpretation is that entrepreneurs are extremely reluctant to be the first to migrate.
} 
distribution, the spatial distribution converges to the initial equilibrium distribution.

\subsection{Agglomeration}

When there is full agglomeration in region $i$, since all the entrepreneurs reside in region $i$, their weighted average utility is $\bar{V}=V_{i}$. Agglomeration is stable if $V_{j}<\bar{V}, \forall j \in N \backslash\{i\}$. The following result gives the condition for stability in parameter space.

Proposition 2. Agglomeration is stable if:

$$
\frac{(1-\phi)[\lambda-(n+\lambda) \phi]}{n \sigma \phi}+\frac{\ln (\phi)}{\sigma-1}<0,
$$

and is unstable if the opposite inequality holds.

Proof. See Appendix B.

Agglomeration is stable if trade costs are sufficiently low. There exists a threshold level $\phi_{s}$, called sustain point, such that agglomeration is stable if $\phi>\phi_{s}$ and unstable if $\phi<\phi_{s}{ }^{8}$ The sustain point $\phi_{s}$ is implicitly defined by:

$$
\frac{\left(1-\phi_{s}\right)\left[\lambda-(n+\lambda) \phi_{s}\right]}{n \sigma \phi_{s}}+\frac{\ln \left(\phi_{s}\right)}{\sigma-1}=0
$$

We can also observe that agglomeration is stable if the immobile to mobile labour ratio is sufficiently high. Rewriting (18), we obtain that agglomeration is stable if $\lambda<\lambda_{s}$, where:

$$
\lambda_{s} \equiv \frac{n \phi[(\sigma-1)(1-\phi)-\sigma \ln \phi]}{(\sigma-1)(1-\phi)^{2}},
$$

and is unstable if $\lambda>\lambda_{s}$.

\subsection{Symmetric dispersion}

The following proposition establishes that full dispersion of entrepreneurs is stable if trade costs are sufficiently high.

Proposition 3. Dispersion is stable if:

$$
\phi<\phi_{b} \equiv \frac{\sigma(1-\lambda)+\lambda}{\lambda+n-\sigma(\lambda+2 n-1)}
$$

\footnotetext{
${ }^{8}$ The left-hand side of $(18)$, denoted $F(\phi)$, has a single zero in the domain $\phi \in(0,1)$. To verify this, check that $\lim _{\phi \rightarrow 0} F(\phi)=+\infty, \lim _{\phi \rightarrow 1} F(\phi)=0, \lim _{\phi \rightarrow 1} F^{\prime}(\phi)>0$, and that $F^{\prime}(\phi)$ has a single zero.
} 
and is unstable if $\phi>\phi_{b}$.

Proof. See Appendix B.

The threshold value $\phi_{b}$ is called break point. ${ }^{9}$ A common assumption in NEG literature is that $\phi_{b}>0$, so that stability of symmetric dispersion cannot be precluded. This is called the no black hole condition and is equivalent to:

$$
\lambda>\frac{\sigma}{\sigma-1}
$$

Since we require consumption of the agricultural good to be positive at full dispersion, it follows that $\lambda>\max \left\{\sigma-1, \frac{\sigma}{\sigma-1}\right\} \cdot{ }^{10}$

Rewriting the stability condition in (21), we find that dispersion is stable if:

$$
\lambda>\lambda_{b} \equiv \frac{\sigma(2 n \phi+1-\phi)-n \phi}{(\sigma-1)(1-\phi)},
$$

and unstable if $\lambda<\lambda_{b}$. Dispersion is thus stable if labour mobility is sufficiently low.

\subsection{Boundary dispersion}

At boundary dispersion, entrepreneurs are evenly distributed among $k \in\{2, \ldots, n-1\}$ regions while the other $n-k$ regions are empty, where $n \geq 3$. By symmetry, we can suppose w.l.o.g. that $h_{j}=0$ for $j=1, \ldots, n-k$ and $h_{j}=\frac{1}{k}$ for $j=n-k+1, \ldots, n$.

Theorem 1. Boundary dispersion is always unstable.

Proof. See Appendix B.

Theorem 1 provides analytical confirmation of the numerical evidence presented by Fujita et al. (1999), Castro et al. (2012), and Gaspar et al. (2013). These authors used CorePeriphery models with 3 or more regions to provide numerical evidence that boundary dispersion with $k=2$ is always unstable. ${ }^{11}$

\footnotetext{
${ }^{9}$ Substituting $n=2$ in (21) we recover the break point of the model of Pflüger (2004).

${ }^{10}$ If $\sigma>\frac{1}{2}(3+\sqrt{5})$ the no black hole condition is implied by $\lambda>\sigma-1$ and thus becomes redundant.

${ }^{11}$ The first two works considered the original CP model (Krugman, 1991), whereas the latter considered the FE model proposed by Forslid and Ottaviano (2003).
} 


\subsection{Partial agglomeration}

\subsubsection{Existence of partial agglomeration equilibria}

In an $n$-region model, there potentially exist several different kinds of asymmetric interior equilibria. We restrict our analysis to a one-dimensional subspace of $\triangle$, defined by $\triangle_{i n v} \equiv$ $\left\{h \in \triangle: h_{j}=\frac{1-h_{1}}{n-1}, \forall j \neq 1\right\}$, which is invariant for the dynamics. This is a particular subspace in a family of one-dimensional subspaces where $k$ regions have the same share of entrepreneurs and the other $n-k$ regions also have the same share of entrepreneurs. We focus on the case where only one region differs from the others in size $(k=1$, or $k=n-1)$. Note that agglomeration $\left(h_{1}=1\right)$ and dispersion $\left(h_{1}=\frac{1}{n}\right)$ are distributions in $\triangle_{i n v}$. The next result is on the maximum number of equilibria in $\triangle_{i n v}$ that are interior and asymmetric, i.e., such that $h_{1} \in(0,1 / n) \cup(1 / n, 1)$.

Proposition 4. There exist at most two interior asymmetric equilibria in $\Delta_{\text {inv }}$ : at most one with $h_{1} \in(0,1 / n)$, whereas at most two with $h_{1} \in(1 / n, 1)$. A distribution $h \in \Delta_{\text {inv }}$ with $h_{1} \in\left(0, \frac{1}{n}\right) \cup\left(\frac{1}{n}, 1\right)$ is an equilibrium if and only if $\lambda=\lambda^{*}\left(h_{1}\right)$, where:

$$
\begin{aligned}
& \lambda^{*}\left(h_{1}\right) \equiv \frac{n(\sigma-1)(1-\phi) \phi\left(h_{1} n-1\right)-n \sigma\left[h_{1}(1-\phi)+\phi\right]\left[\phi\left(h_{1}+n-2\right)-h_{1}+1\right] \nu\left(h_{1}\right)}{(\sigma-1)(1-\phi)^{2}\left(h_{1} n-1\right)}, \\
& \text { with } \nu\left(h_{1}\right) \equiv \ln \left\{\frac{\phi\left(h_{1}+n-2\right)-h_{1}+1}{(n-1)\left[h_{1}(1-\phi)+\phi\right]}\right\} .
\end{aligned}
$$

Proof. See Appendix B.

Note that $h_{1} \in(1 / n, 1)$ means that region 1 has more industry than the other regions and in this case we refer to region 1 as a partial core.

We illustrate in Figure 1 the possible multiplicity of partial agglomeration equilibria, drawing $\lambda^{*}\left(h_{1}\right)$ for $n=3$ and $\sigma=4$, for two different values of $\phi$.

When $\phi$ is low (picture to the left), there may exist one, two or zero interior asymmetric equilibria in $\triangle_{i n v}$. For $\lambda_{A}<\lambda<\lambda_{B}$, there is only one equilibrium and it does not have a partial core. For $\lambda_{B}<\lambda<\lambda_{C}$, there are two equilibria and at least one has a partial core (both have if $\lambda$ is relatively high). If $\lambda>\lambda_{C}$, there are no equilibria. When $\phi$ is high (picture to the right), there is at most one interior asymmetric equilibria in $\triangle_{i n v}$. If $\lambda$ is high, it has a partial core.

\subsubsection{Stability of partial agglomeration}

At a partial agglomeration equilibrium, two types of migrations can take place. One migration concerns movements along the invariant space $\triangle_{i n v}$, i.e., from region 1 to the evenly populated regions. If $n-1$ entrepreneurs leave region 1 , each of the other regions 

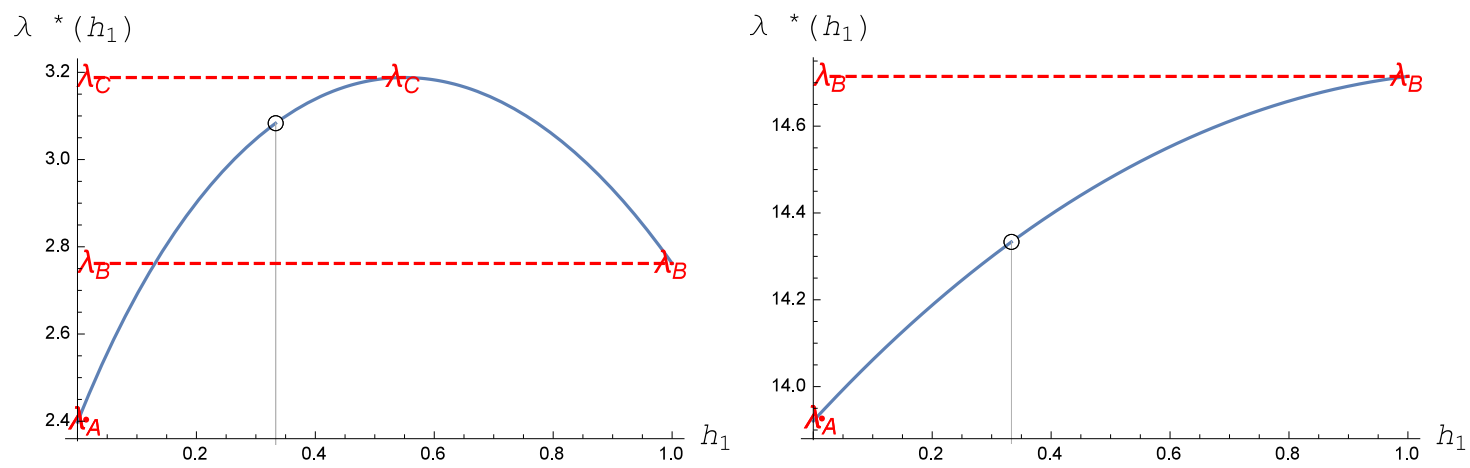

Figure 1 - Illustration of $\lambda^{*}\left(h_{1}\right)$ for $n=3$. On the vertical axis we present values of $\lambda$ such that $h_{1} \in(0,1) \backslash\{1 / 3\}$ is an interior equilibrium. To the left, we have $\phi=0.2$ and to the right we have $\phi=0.65$. The equilibria occur at the intersection of a horizontal line ( $\lambda$ constant) with the lines depicted in each figure.

will get 1 of those entrepreneurs. Since, along the invariant space, regions $\{2, \ldots, n\}$ share the same size, the decisions of these $n-1$ entrepreneurs are equivalent. The other migration concerns that of an entrepreneur who chooses to move exogenously between two regions other than region 1 (transversally to $\triangle_{i n v}$ ). We have the following result.

Theorem 2. Partial agglomeration is stable if $h_{1} \in(1 / n, 1)$ and:

$$
\delta\left(h_{1}\right) \equiv(1-\phi)\left(h_{1} n-1\right)[(n-1) \phi+1]+\Phi\left(h_{1}\right) \nu\left(h_{1}\right)<0,
$$

where $\Phi\left(h_{1}\right)=h_{1}^{2} n(1-\phi)^{2}-2 h_{1}(1-\phi)^{2}+\phi\{n[(n-3) \phi+2]+3 \phi-4\}+1$ and $\nu\left(h_{1}\right)$ is as in (24). It is unstable if either $h_{1} \in(0,1 / n)$ or $\delta\left(h_{1}\right)>0$.

Proof. See Appendix B.

Partial agglomeration can only be stable if the asymmetric region is a partial core. If this is the case, stability depends solely on the effect of migration on utilities between a partial core and any of the other regions, i.e., on $\delta$. Notice that $\nu\left(h_{1}\right)<0$ and $\Phi\left(h_{1}\right)>0$ if $h_{1}>$ $1 / n$. Therefore, the term $\Phi\left(h_{1}\right) \nu\left(h_{1}\right)<0$ constitutes the agglomerative force attracting entrepreneurs towards the partial core, whereas $(1-\phi)\left(h_{1} n-1\right)[(n-1) \phi+1]>0$ is the dispersive force that drives entrepreneurs away from the partial core to the other evenly populated regions.

To understand why partial agglomeration is unstable if $h_{1} \in(0,1 / n)$, notice that a small exogenous migration from region $n$ to region $j \neq 1$ decreases the cost-of-living in region $j$ due to the increase of the number of varieties produced locally. This is captured by differentiating the second term of $V_{j}$ in (16) and evaluating at partial agglomeration, which gives:

$$
\frac{\mu(n-1)(1-\phi)}{(\sigma-1)\left[\phi\left(h_{1}+n-2\right)-h_{1}+1\right]}>0 .
$$




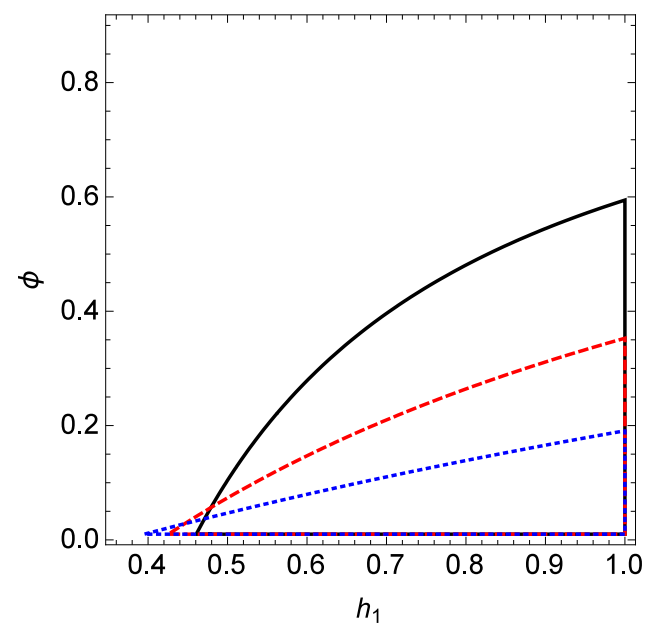

Figure $2-$ Regions of stability $\delta_{n}<0$ in $(h, \phi)$ space, for $n=\{3,5,10\}$. The region below the solid line corresponds to $\delta_{3}<0$; below the dashed line we have $\delta_{5}<0$; the dotted line contain $\delta_{10}<0$.

On the other hand, the nominal wage in region $j$ decreases:

$$
\left.\frac{\partial w_{j}}{\partial h_{j}}\left(h_{1}, \frac{1-h_{1}}{n-1}\right)\right|_{\lambda=\lambda^{*}\left(h_{1}\right)}=\frac{(n-1)^{2} \sigma\left[h_{1}(1-\phi)+\phi\right] \nu\left(h_{1}\right)}{(\sigma-1)\left(h_{1} n-1\right)\left[\phi\left(h_{1}+n-2\right)-h_{1}+1\right]},
$$

which is negative for $h_{1} \in(0,1 / n)$. When $h_{1} \in(0,1 / n)$, the cost-of-living effect always outweighs the dispersive decrease in nominal wage. The receiving region, having become the largest after migration, will tend to further develop into an industrialized core. This explains why it is not possible for industry to spread evenly among $n-1$ regions when the other region is less industrialized.

Stability of partial agglomeration requires a significantly industrialized partial core. There exists $\epsilon>0$ such that partial agglomeration is unstable if $h_{1} \in\left(\frac{1}{n}, \frac{1}{n}+\epsilon\right) .{ }^{12}$ Stability of partial agglomeration also requires that trade costs are not too low. There exists $\epsilon>0$ such that partial agglomeration is unstable for $\phi \in(1-\epsilon, 1) .{ }^{13}$ In order to convey a better picture, we illustrate in Figure 2 the region $\delta<0$ in $\left(h_{1}, n\right)$ space, for $n=\{3,5,10\}$. The numerical evidence suggests that, with more regions, partial agglomeration requires higher barriers to trade (lower $\phi$ ). With more regions, some partial agglomeration equilibria may arise with less industry in the partial core because total dispersion implies a lower share of entrepreneurs in each region. Finally, Figure 2 shows that partial agglomeration with $h_{1} \in(1 / n, 1)$ is stable if the freeness of trade, $\phi$, is low enough. The more industrialized the partial core is, the higher is the range of freeness of trade values for

\footnotetext{
${ }^{12}$ This stems from the fact that $\delta\left(h_{1}=1 / n\right)=0, \frac{\partial \delta}{\partial h}(h=1 / n)=0$ and $\frac{\partial^{2} \delta}{\partial h^{2}}(h=1 / n)>0$.

${ }^{13}$ This is a consequence of $\delta(\phi=1)=0, \frac{\partial \delta}{\partial \phi}(\phi)=0$ and $\frac{\partial^{2} \delta}{\partial \phi^{2}}(\phi=1)>0$.
} 
which partial agglomeration is a stable equilibrium.

\section{Bifurcations in the $n$-region model}

Most 2-region NEG models under exogenous symmetry undergo pitchfork bifurcations at the symmetric dispersion when the parameter path concerns smooth changes only in transportation costs. Pflüger and Südekum (2008a) have shown that changes in the functional form of the utility function produce modifications in the qualitative structure of a class of footloose-entrepreneur models. However, these changes affect the type of pitchfork bifurcation rather than the type of bifurcation itself. ${ }^{14}$ For instance, the 2-region QL (Pflüger, 2004) undergoes a supercritical pitchfork bifurcation at symmetric dispersion. We next show that when more than 2 regions come to interplay, the QL model exhibits instead a primary transcritical bifurcation at the symmetric equilibrium and a secondary saddle-node bifurcation that branches from an interior asymmetric equilibrium. A distinctive feature of these bifurcations is that the qualitative change in spatial distributions as transport costs decrease here is not reflected symmetrically across all regions, even though the model is completely symmetric in all respects. This suggests that the role of transport costs in an equidistant multi-regional model may be more complex than what is envisaged in most agglomeration models. ${ }^{15}$

The remainder of the section is dedicated to explaining the details of these bifurcations and their consequences on the qualitative transition towards more agglomerated distributions as trade integration increases.

\subsection{Primary and secondary bifurcations}

We have the following results.

Proposition 5. In the $n$-region $(n \geq 3)$ QL model symmetric dispersion undergoes a transcritical bifurcation at the break point.

Proof. See Appendix C.

From this result, symmetric dispersion looses stability as $\phi$ rises above $\phi_{b}$. A primary branch of partial agglomeration equilibria crosses symmetric dispersion at the bifurcation

\footnotetext{
${ }^{14}$ In the model of Ottaviano et al. (2002), the resulting bifurcation is a borderline case between a supercritical and subcritical pitchfork.

${ }^{15}$ Bifurcation in core-periphery models has been addressed by Berliant and Kung (2009) in a different context. The variety of bifurcations is obtained through the addition of parameters to the original model.
} 
point $\phi=\phi_{b}$. For $\phi<\phi_{b}$, this branch lies in the region $h_{1} \in(1 / n, 1)$. For $\phi>\phi_{b}$, the branch lies in the region $h_{1} \in(0,1 / n)$. Locally, both before and after the bifurcation occurs, the partial agglomeration equilibria along the primary branch are unstable in a neighbourhood of the bifurcation point. ${ }^{16}$

In order to understand the behaviour of partial agglomeration equilibria on the entire invariant space $\Delta_{i n v}$ for $h_{1} \in(1 / n, 1)$, we take a further step by verifying the conditions for a secondary bifurcation along the primary branch that occurs at a fold point, $\phi_{f}$. Because we are looking at the invariant subspace $\left(h_{1}, h_{j}\left(h_{i}\right)\right)=\left(h_{1},\left(1-h_{1}\right) /(1-n)\right)$, the study of bifurcations at the symmetric dispersion equilibrium in this restriction of the $n$-region model is reduced to a one dimensional case.

Proposition 6. Along the primary branch for $h_{1} \in(1 / n, 1)$, the $n$-region $Q L$ model undergoes a saddle-node bifurcation as $\phi$ decreases from $\phi_{b}$.

\section{Proof. See Appendix C.}

The existence of a secondary saddle-node bifurcation for $h_{1} \in(1 / n, 1)$, together with the direction of the transcritical bifurcation and stability of its branches, ensures that $\phi_{f}<\phi_{b}$ and $\phi_{f}<\phi_{s}$. As $\phi$ increases above $\phi_{f}$, two partial agglomeration equilibria appear. The one with the more industrialized partial core is stable, whereas the other one is unstable. In other words, the saddle-node bifurcation is characterized by a curve of partial agglomeration equilibria along a primary branch for $h_{1} \in(1 / n, 1)$ that is tangent to the line $\phi=\phi_{f}$ and lies to its right. ${ }^{17}$

One important question concerns whether $\phi_{b}<\phi_{s}$ or $\phi_{b}>\phi_{s}$. The relative position of these thresholds determines the smoothness of the progressive industrialization process as the freeness of trade increases. On the other hand, the qualitative structure of the model ensures that there always exists a stable spatial distribution with $h_{1} \in[1 / n, 1]$, for every freeness of trade value $\phi \in(0,1)$.

We first focus on the case $\phi_{b}<\phi_{s}$, which requires a relatively low $\lambda$. Figure 3 depicts the QL model's bifurcation diagram in the invariant space $\triangle_{i n v}$ for $n=3$. For the illustrations we set $\mu=0.3, \sigma=4$ and $\lambda=2.5 .^{18}$ The interpretation strays from that of the typical bifurcation diagrams of 2-region models in NEG literature, because we are restricting ourselves to the invariant subspace $\triangle_{i n v} \subset \triangle$. Just like the simplex in the

\footnotetext{
${ }^{16}$ The branch for $h_{1} \in(0,1 / n)$ is stable (only) along the invariant space. From Theorem 2 , however, it is unstable.

${ }^{17}$ The details that support these claims about the bifurcations are provided by the derivatives in (T3), (T4), and (SN3) in Appendix C.

${ }^{18}$ The parameter values chosen for the simulations ensure that $w_{i}, w_{j}>\mu$ at every partial agglomeration equilibrium, so that entrepreneurs consume both goods at every possible distribution.
} 


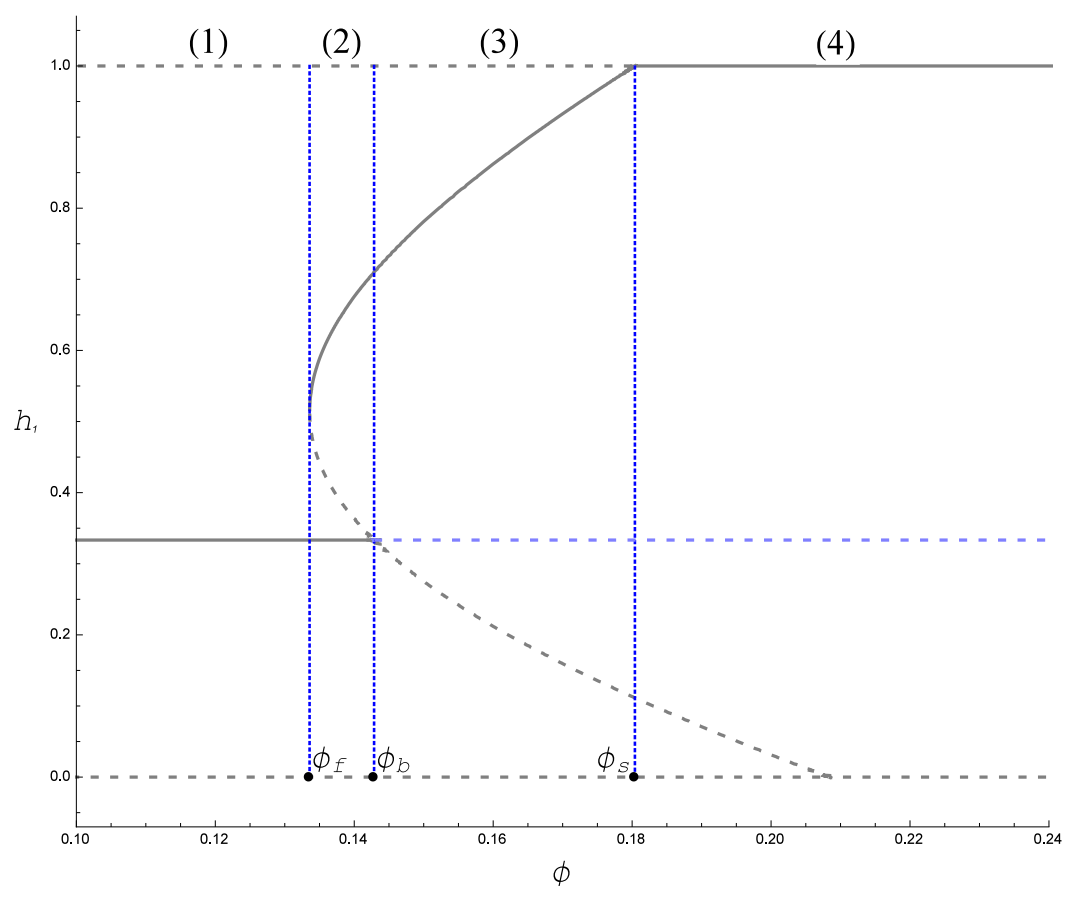

Figure 3 - Bifurcation diagram for the 3-region model. Solid and dashed lines represent stable and unstable equilibria, respectively. Vertical dashed lines delimit areas (1) to (4) as follows: (1) stability of symmetric dispersion; (2) stability of both dispersion and partial agglomeration; (3) stability of partial agglomeration; (4) stability of full agglomeration.

2-region model, the subspace is also one dimensional here and migration movements that affect region 1 will affect all other regions. A migration from (to) region 1 would result in $2 x$ entrepreneurs leaving (entering) region 1 for $x$ entrepreneurs that enter (leave) each of the other 2 regions. ${ }^{19}$

It thus comes as no surprise to see that $h_{1}=0$ corresponds to a qualitatively different spatial distribution when $n=2$ and when $n \geq 3$. When $n=2$, it corresponds to agglomeration. When $n \geq 3$, it corresponds to border dispersion, which is never stable.

In figure 3 we can see the primary transcritical bifurcation branching from the break point $\phi_{b}$ and the secondary saddle-node bifurcation occurring along the primary branch, whose fold point, $\phi_{f}$, is located in the upper part of the invariant subspace. Notice that an initially partially agglomerated industry will be forced to fully disperse across regions if there is a decrease in the freeness of trade below $\phi_{f}$. If this decrease is temporary, the industry will remain fully dispersed, so there are permanent effects, which means that the $n$-region QL model exhibits locational hysteresis. Formally, this happens because $\phi_{f}<\phi_{b}$.

\footnotetext{
${ }^{19}$ In a 3-region model, along the invariant space, region 1 has $h_{1}$ entrepreneurs and regions 2 and 3 have $h_{2}=h_{3}=\left(1-h_{1}\right) / 2$.
} 

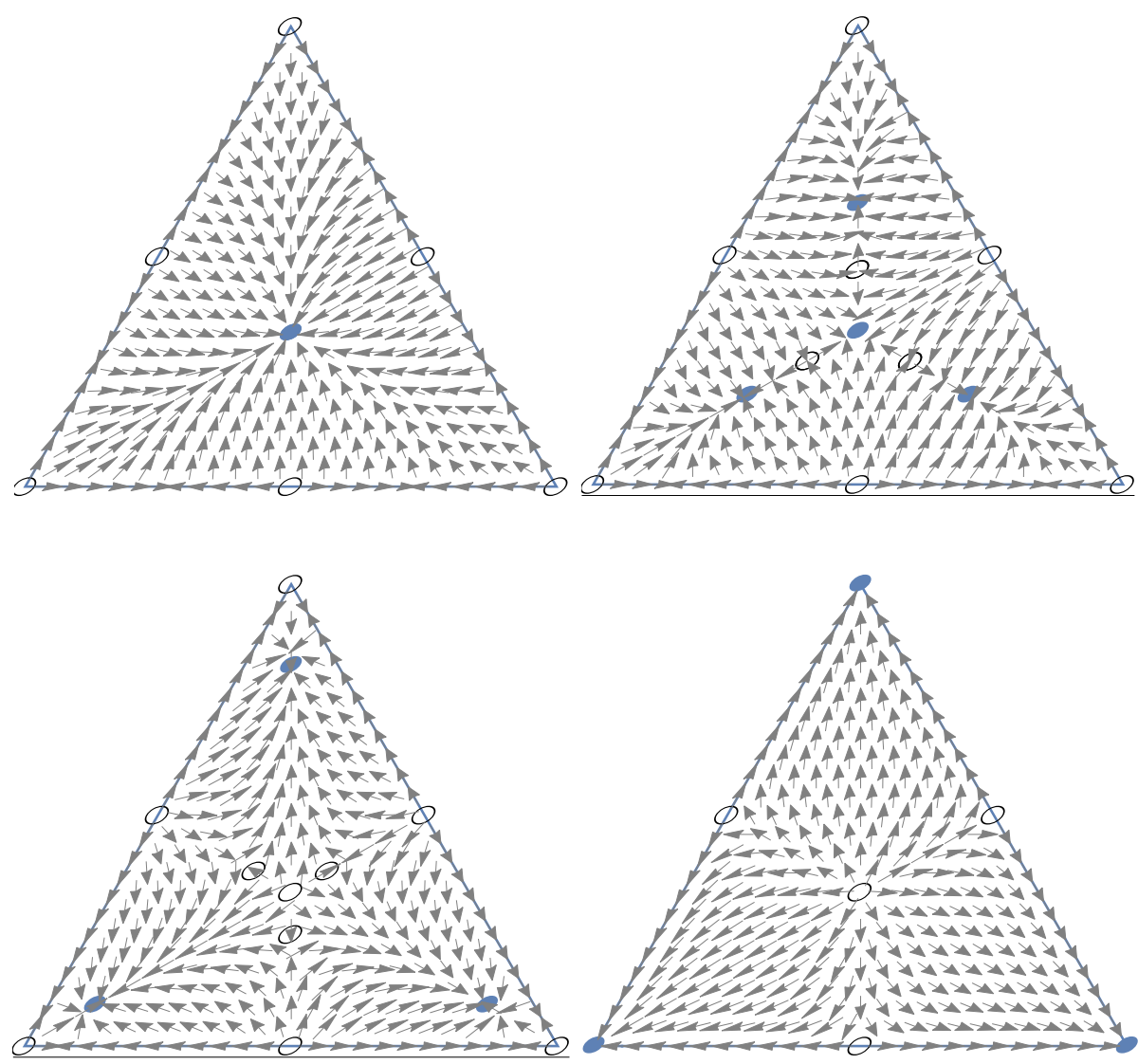

Figure 4 - Migration dynamics of entrepreneurs inside the 2-simplex. From left to right to bottom, $\phi$ is increasing, and each picture corresponds to a region in Figure 3 indexed from (1) to (4). In the first picture, only symmetric dispersion is stable. In the second, both partial agglomeration and symmetric dispersion are stable. In the third picture only partial agglomeration is stable. In the last picture, agglomeration is the only stable equilibrium. 
Figure 4 contains the different dynamics of the 3-region model inside the 2-dimensional simplex, with correspondence to the regions (1)-(4) of Figure 3. We start with low levels of $\phi$. When the freeness of trade is very low, we have $\phi<\phi_{f}<\phi_{b}<\phi_{s}$, so only dispersion is stable (upper left picture). However, once $\phi_{f}<\phi<\phi_{b}<\phi_{s}$, two partial agglomeration equilibria immediately arise on the upper part (for $h_{1} \in(1 / 3,1)$ ) of the invariant space $\triangle_{i n v}$ of the simplex (upper left picture). The one that lies closer to agglomeration is the only stable equilibrium. In the bottom left picture, $\phi$ has risen just above the break point but lies just below the sustain point, i.e., $\phi_{f}<\phi_{b}<\phi<\phi_{s}$. At this point there are still two partial agglomeration equilibria; however, one of them lies in the inferior part of the invariant space $\triangle_{i n v}$ and is unstable whereas the other is stable. As $\phi$ approaches the sustain point, the stable partial agglomeration equilibrium approaches agglomeration until it disappears once $\phi>\phi_{s}$, after which agglomeration becomes the only stable spatial distribution.

Intuitively, the absence of income effects mitigates agglomeration forces as transport costs decrease, which justifies the existence of partial agglomeration just as in the 2region model. On the other hand, higher market access variability due to the presence of more regions implies that lower transport costs enhance the relative strength between centripetal and centrifugal forces, resulting in a spatial distribution where one region is considerably more industrialized. Further decreases in transport costs then lead to a progressive and smooth transition towards full agglomeration.

We now focus on the case whereby $\phi_{b}>\phi_{s}$. Note that, in Pflüger's 2-region QL model, the existence of a supercritical bifurcation at the break point $\phi_{b}$ precludes this scenario. For $n \geq 3$, we have the following result.

Proposition 7. There exists $a \lambda \in\left(\lambda_{b}, \lambda_{s}\right)$ such that agglomeration and dispersion are both stable if:

$$
n>n_{T} \equiv-\frac{(1-\phi)^{2}}{\phi(1-\phi+\ln \phi)},
$$

Proof. See Appendix C.

The derivatives of $\lambda_{b}$ in (23) and $\lambda_{s}$ in (20) with respect to $\phi$ are both positive. Therefore, a higher freeness of trade increases the range of values of $\lambda$ for which agglomeration is stable and decreases the range of values of $\lambda$ for which dispersion is stable. However, the difference $\lambda_{s}-\lambda_{b}$ can be shown to be increasing in $\phi$. This implies that a higher freeness of trade increases the range of values of immobile to mobile labour ratio for which agglomeration and dispersion are simultaneously stable. Accordingly, $n_{T}$ in (27) is decreasing in $\phi$, which means that simultaneity of stability requires a lower number of regions when the freeness of trade is higher. 


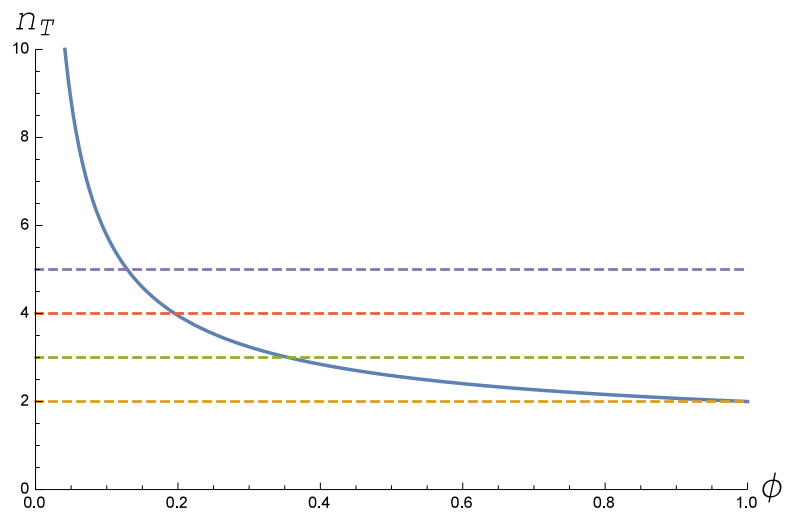

Figure 5 - Threshold $n_{T}$ in (27). Agglomeration and dispersion are simultaneously stable in the graph above the thick line. For $n \geq 3$, simultaneity requires higher transport costs for a higher number of regions.

Figure 5 illustrates $n_{T}$ from (27) in $\phi$ space. It shows that simultaneity of stability of agglomeration and dispersion is not possible in the 2-region model. It also shows that an $(n+1)$-region model favours simultaneity of agglomeration and dispersion for a wider range of transport cost values compared to an $n$-region model.

Corollary 1. For a sufficiently high $\lambda$, there exists a range of transport cost values for which agglomeration and dispersion are both stable.

Proof. If condition (27) holds, then, for some $\lambda$, agglomeration and dispersion are simultaneously stable. Since stability of both equilibria requires $\phi>\phi_{s}$ and $\phi<\phi_{b}$, then it follows that, for some $\lambda$, we must forcibly have $\phi_{b} \geq \phi_{s}$. Moreover, we have $d\left(\phi_{b}-\phi_{s}\right) / d \lambda>0$, which means that $\phi_{b}-\phi_{s}>0$ occurs for a sufficiently high $\lambda$.

We now proceed to illustrate the qualitative structure of spatial distributions when $\phi_{s}<$ $\phi_{b}$. We increase the ratio of immobile to mobile workers compared to the previous simulations, by setting $\lambda=6$. The resulting bifurcation diagram is now presented in Figure 6. Clearly, the main qualitative difference compared to Figure 3 pertains to the region indexed by (3), where we now have $\phi \in\left(\phi_{s}, \phi_{b}\right)$.

Figure 7 portrays the 2-dimensional simplex for $\phi_{b}>\phi_{s}$ once $\phi$ rises above the sustain point (corresponding to region (3) in Figure 6).

We can observe that for $\phi_{s}<\phi<\phi_{b}$, agglomeration and total dispersion are both simultaneously stable, while a single unstable partial agglomeration equilibrium for $h \in$ $(1 / 3,1)$ exists in $\triangle_{i n v}$ between them.

We now sum up the implications of the relative position between the break point and sustain point. If $\phi_{b}<\phi_{s}$, once symmetric dispersion loses stability, a significant migration will occur to a partially agglomerated equilibrium. This migration will be followed by a 


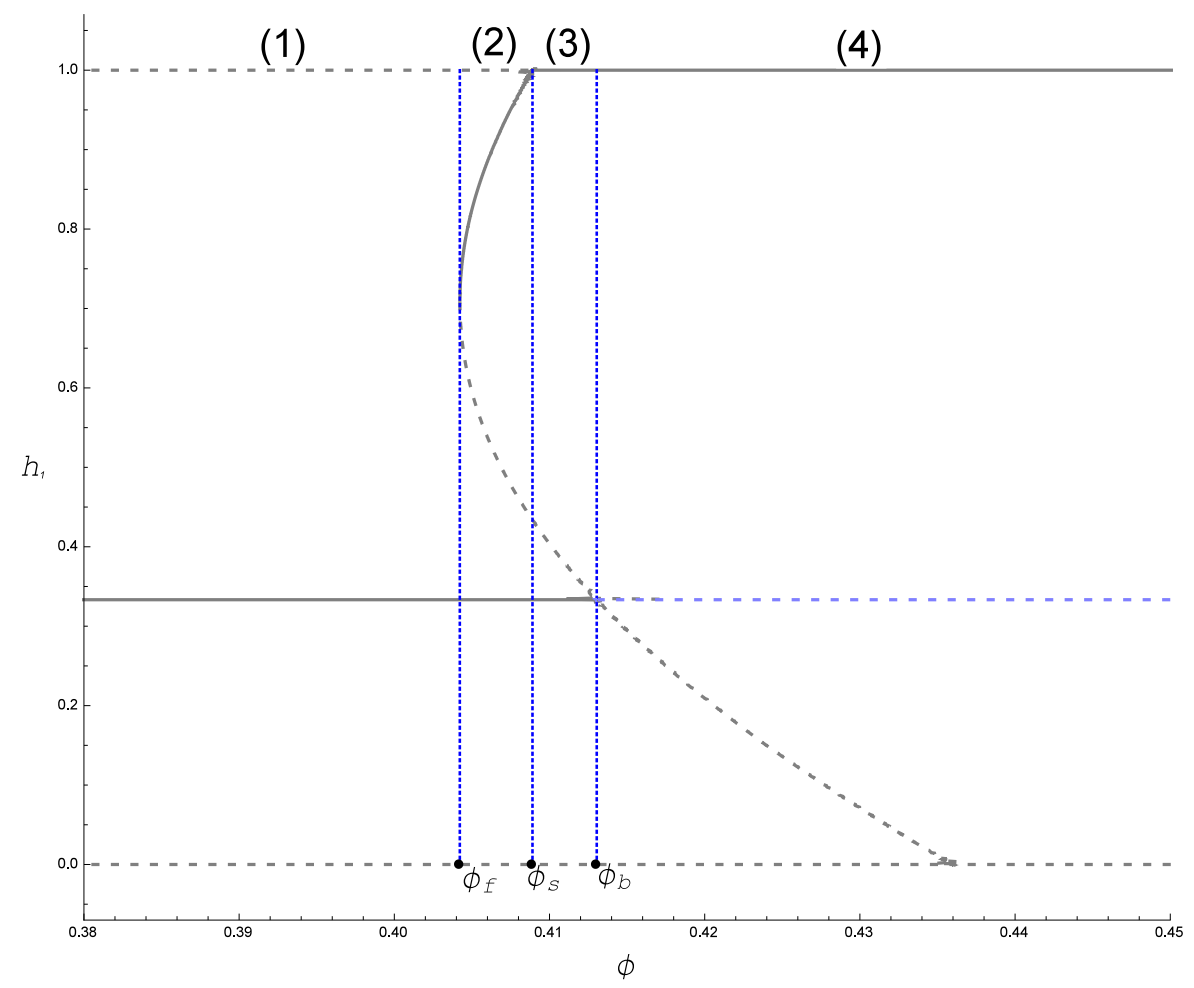

Figure 6 - Bifurcation diagram for the 3-region model. Solid and dashed lines represent stable and unstable equilibria, respectively. Vertical dashed lines delimit areas (1) to (4) as follows: (1) stability of symmetric dispersion; (2) stability of both dispersion and partial agglomeration; (3) stability of both dispersion and full agglomeration; (4) stability of full agglomeration. Parameter values are $\sigma=5$ and $\lambda=6$.

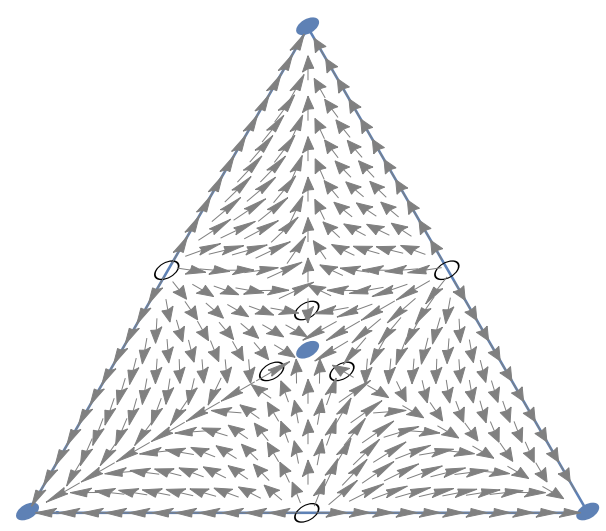

Figure 7 - A 2-simplex portraying for $\phi_{s}<\phi<\phi_{b}$. We can see that agglomeration and symmetric dispersion are both stable. A single unstable partial agglomeration equilibrium exists in the upper part of $\triangle_{i n v}$. 
smooth transition towards agglomeration if $\phi$ rises further. This scenario is illustrated in figures 3 and 4 . Conversely, if $\phi_{b}>\phi_{s}$, entrepreneurs will immediately agglomerate in one single region once dispersion becomes unstable. Moreover, if the increase in $\phi$ above $\phi_{b}$ is due to some temporary policy, agglomeration is permanent. This is depicted in figure 6 . Smoother transitions require a lower immobile to mobile labour ratio $\lambda$, whereas catastrophic agglomeration is more likely under higher values of $\lambda^{20}$

In economic terms, when overall inter-regional mobility is low, dispersion forces are higher because firms have larger incentives to relocate to less industrialized regions in order to capture local demand and avoid fiercer competition in more crowded markets. However, dispersion forces due to existence of immobile workers in other markets are naturally stronger at more symmetric distributions. The implication is that a lower global inter-regional mobility encourages symmetric dispersion more than it discourages full agglomeration. When it is too low, if agglomeration forces exceed dispersion forces at symmetric dispersion, the spatial distribution then immediately shifts towards full agglomeration.

\subsection{A note on the black hole condition and on the role of inter- regional mobility}

We know that there is a condition on which stability of total dispersion hinges crucially; the no black hole condition. In early NEG literature, the no black hole condition may have been a requirement assumed out of necessity or convenience; after all, in a fully symmetric two region, two industry setting, precluding dispersion would doom geography to the unlikely prediction of full agglomeration in one region. In our context, the no black hole condition would seem ad hoc to say the least.

Figure 8 shows a bifurcation diagram where the ratio of inter-regionally immobile workforce relative to mobile (skilled) labour is lower than unity and, as such, total dispersion is precluded. The results concerning bifurcations in the previous section do not extend to this case since we know that $\phi_{f}<\phi_{b}$. In fact, as the freeness of trade increases, the spatial distribution approaches agglomeration monotonically from an interior asymmetric equilibrium along the invariant space $\triangle_{i n v}$.

On account of these findings, we conclude that a higher global inter-regional worker mobility (lower $\lambda$ ) leads to smoother transitions towards agglomeration as transport costs steadily decrease.

\footnotetext{
${ }^{20}$ Our results can be shown to extend to a fairly general range of values for $\lambda$.
} 


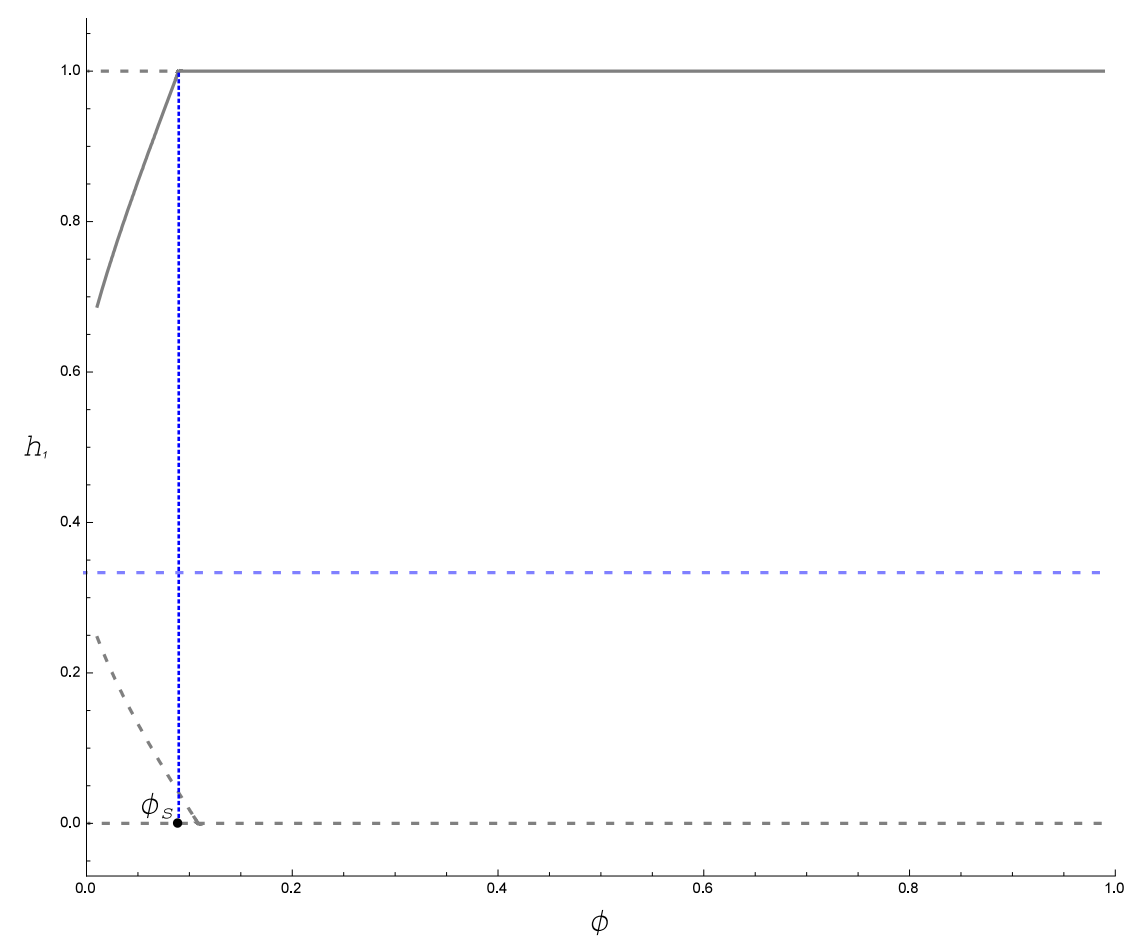

Figure 8 - If the unskilled labour force is relatively low, dispersion is not a stable outcome, but partial agglomeration is still possible. We have $\sigma=2.5$ and $\lambda=1.6$.

\section{Welfare}

We have analysed the different possible spatial distributions in the the QL model with an arbitrary number of equidistant regions. We now analyse the desirability of these possible distributions. For normative purposes, we adopt a utilitarian criterion similar to that of Pflüger and Südekum (2008b), thus looking at the average indirect utility of entrepreneurs, farmers, and then at the whole economy.

\section{$5.1 \quad$ Entrepreneurs}

We start by showing how the spatial distribution of entrepreneurs affects their well-being.

Theorem 3. The average utility of entrepreneurs is convex in the spatial distribution of entrepreneurs $h$, attaining a global minimum at symmetric dispersion.

Proof. See Appendix D.

Total dispersion is the spatial distribution that yields the worst outcome for the entrepreneurs as measured by average utility. To understand why entrepreneurs may be driven to the situation that minimizes their overall welfare, it is useful to consider an economy with 3 regions whose distribution is initially fully dispersed. Supposing that full dispersion is 
stable, consider a marginal exogenous exodus from region 1 to region 3, which increases average utility (from Theorem 3). However, since dispersion is stable, entrepreneurs in region 3 will now observe that $V_{1}>\bar{V}$. Since they are short-sighted and move to the region with the highest utility, they will return to region 1 , restoring symmetric dispersion. This situation is similar to the standard prisoner's dilemma. As noticed by Pflüger and Südekum (2008b), the continuum of stable equilibria with partial agglomeration that the absence of income effects allows for, as opposed to other NEG models, may contribute to this result. Adding dispersion forces such as a housing sector (Pflüger and Südekum, 2008b) or commuting costs such as in Ottaviano et al. (2002) and Tabuchi et al. (2005) would most likely improve the welfare of entrepreneurs at less agglomerated outcomes.

\section{$5.2 \quad$ Farmers}

Since the nominal wage of farmers is normalised to 1 , all changes in their welfare are caused by changes in average price indices. ${ }^{21}$ From (15), their average utility is given by:

$$
\bar{V}^{L}(h)=1+\frac{\mu}{n(\sigma-1)} \sum_{j=1}^{n} \ln \left[\phi+(1-\phi) h_{j}\right]+\eta .
$$

In contrast with entrepreneurs, farmers prefer more dispersed distributions.

Theorem 4. Farmers' average indirect utility is concave in the spatial distribution of entrepreneurs $h$, attaining a global maximum at symmetric dispersion.

Proof. See Appendix D.

Farmers attain the highest welfare when all entrepreneurs are evenly dispersed among all regions. Regional indirect utility of farmers in region $i$ is strictly increasing in the number of entrepreneurs that reside there, due to the fact that locally produced varieties are sold at lower prices. ${ }^{22}$ Therefore, industrialization of a region leads to a progressive improvement of the welfare of farmers residing in that region. However, on average, farmers as a whole become relatively poorer.

\subsection{Social welfare}

The results shown so far evidence a clear trade-off between the welfare of entrepreneurs and the welfare of farmers. For a given stable equilibrium, the welfare of the economy as

\footnotetext{
${ }^{21}$ Recalling Proposition 1, a similar statement applies to the average welfare of entrepreneurs.

${ }^{22}$ In fact, it is possible to rewrite $P_{i}$ in a way that it depends only on $h_{i}$. See proof of Theorem 3.
} 
a whole thus depends on the global ratio of farmers to entrepreneurs, $\lambda$, and the number of regions $n$. Let us define social welfare as an average of both average indirect utilities that depends on the spatial distribution of entrepreneurs:

$$
\Omega(h)=\frac{1}{\lambda+1}\left[\bar{V}(h)+\lambda \bar{V}^{L}(h)\right] .
$$

Rewriting (29) using (28) and $\bar{V}$ we get:

$$
\Omega(h)=\frac{1}{\lambda+1}\left\{\varepsilon+\frac{\mu}{(\sigma-1)}\left[\sum_{j=1}^{n} \ln \left[\phi+(1-\phi) h_{j}\right]\left(\frac{\lambda}{n}+h_{j}\right)\right]\right\},
$$

where $\varepsilon=\lambda(1+\eta)+\bar{w}+\eta$ is a constant. The next result provides valuable information concerning the local extrema of $\Omega(h)$.

Proposition 8. Social welfare interior extrema are located on one-dimensional invariant spaces whereby $k$ regions have a share of entrepreneurs $h_{i}=h_{1} / k$ and the other $n-k$ regions have a share $h_{j}=\left(1-h_{1}\right) /(n-k)$.

Proof. See Appendix D.

As we have seen before, one particular invariant space of this kind is $\Delta_{i n v}$, which corresponds to the particular case where $k=1$ or $k=n-1$. From Proposition 5, a Corollary follows for the 3-region case.

Corollary 2. For $n=3$, all social welfare interior extrema are located on $\Delta_{i n v}$.

Proof. In the 3-region model, the only invariant spaces that correspond to the ones identified in Proposition 5 are the three invariant spaces whereby one region has $h_{1}$ entrepreneurs and the other two regions have $h_{j}=h_{1} / 2$. This matches the invariant space $\Delta_{i n v}$.

From Proposition 5, the study of social welfare interior extrema can be reduced to the simpler one-dimensional invariant space. Additionally, Corollary 2 ensures that in order to study welfare in a 3-region model we need only look at the one-dimensional subspace $\Delta_{i n v}$ in addition to the boundaries. ${ }^{23}$ Using (30) and $n=3$, we find:

$$
\Omega\left(h_{1}\right)=\frac{1}{3(\lambda+1)}\left[3 \varepsilon+\frac{\mu}{(\sigma-1)} \zeta\right]
$$

\footnotetext{
${ }^{23}$ For $n \geq 4$, there may exist other potential extrema on other invariant spaces (as well as equilibria).
} 
where:

$$
\zeta=\left(3 h_{1}+\lambda\right) \ln \left[h_{1}(1-\phi)+\phi\right]+\left[2 h+3\left(1-h_{1}\right)\right] \ln \left[\frac{1-h_{1}}{2}(1-\phi)+\phi\right] .
$$

Figure 9 plots the different possible shapes of social welfare for increasing levels of $\phi$, for $\lambda=3$ and $\sigma=5$. When $\phi$ is low (upper left picture), symmetric dispersion is the only stable equilibrium and is a global welfare maximum. For a slightly higher $\phi$ (upper right picture), both dispersion and a highly industrialised partial agglomeration dispersion are stable but the former is still a global welfare maximum. Increasing $\phi$ further (medium left picture) makes dispersion unstable, whereas partial agglomeration is stable but is socially inferior compared to any less agglomerated spatial distribution. As $\phi$ rises even further (medium right picture) agglomeration becomes stable but is dominated by another less asymmetric distribution. However, continuous increases in $\phi$ steadily improve the welfare at agglomeration until it eventually becomes a global welfare maximum (lower pictures). The evidence here shows that the 3-region model exhibits a tendency towards over-agglomeration for intermediate transport cost levels.

In order to check if this tendency carries over to the general $n$-region model, we present the following Theorem.

Theorem 5. From a social point of view: (i) if dispersion is stable, it is a local maximum and always superior to agglomeration; (ii) there exists $\phi_{z} \in\left(\phi_{s}, 1\right)$ such that agglomeration is stable for $\phi \in\left(\phi_{s}, \phi_{z}\right)$, but is socially inferior to other less asymmetric distributions.

\section{Proof. See Appendix D.}

From Theorem 5 we learn that there is a range for the freeness of trade just above the sustain point for which agglomeration is a stable outcome, but also whereby social welfare is lower compared to other less industrialized distributions. Therefore, there is a tendency towards over-agglomeration. This is particularly notorious if $\phi_{b}>\phi_{s}$, because a temporary increase in the freeness of trade just above $\phi_{b}$ permanently shifts the spatial distribution to a socially inferior outcome. ${ }^{24}$

\footnotetext{
${ }^{24}$ On the other hand, it can be shown that a higher worker mobility (lower $\lambda$ ) and a higher trade freeness increases the likelihood that global welfare will be greater at agglomeration compared to dispersion. This statement is true if $\lambda>n \phi /(1-\phi)$, which we assume; otherwise agglomeration is always stable (see equation (18) in Section 3.1).
} 

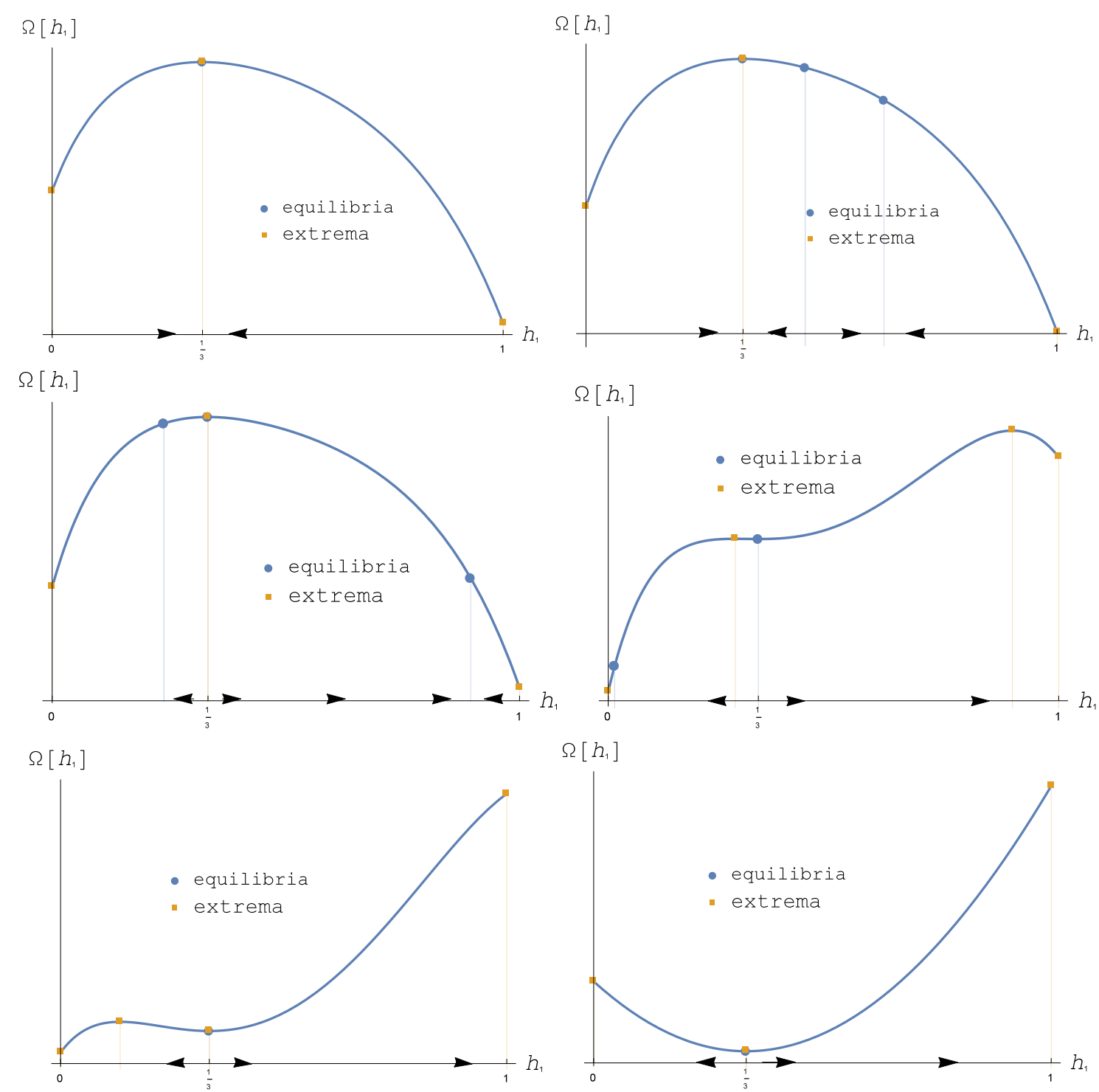

Figure 9 - Welfare along the invariant space $\Delta_{i n v}$ for the 3-region model (with $\lambda=3$ and $\sigma=5$ ). From left to right to bottom, $\phi$ is increasing. 


\section{Conclusion}

The QL model presents itself as a good candidate for extending the study of NEG to a higher number of regions. By considering a quasi-linear upper tier utility function, the absence of income effects on consumers' demand for manufactures enables one to obtain simpler analytical expressions for the entrepreneurs' real wages in each region. In most other NEG models with different utility functional forms, regional wages feed back on the entrepreneurs' incomes in all other regions, which in turn depend on the wages they get, making it progressively harder to obtain tractable expressions as more regions are considered.

As we have seen, the QL model allows to study NEG with an arbitrary number of equidistant regions under exogenous symmetry. Moreover, it accommodates for the possibility of partial agglomeration equilibria, a feature which is ruled out in many CP models under exogenous symmetry. This enforces the idea that exogenous asymmetries are not the only source of asymmetric spatial distributions.

We look at equilibria where at least one region is absent of industry and the remaining regions are evenly industrialized, and find they are always unstable. To the best of our knowledge, this is the first analytical confirmation that an evenly distributed industry among less than the total number of regions is not possible.

We look at partial agglomeration distributions along invariant spaces where all but one region share the same level of industry. Contrary to the 2-region QL model (Pflüger, 2004), where the only invariant space is the entire set of spatial distributions itself and any distribution may correspond to a stable equilibrium, with three and more regions, along the aforementioned invariant space, a partial agglomeration equilibrium can only be stable if a single region has a relatively larger industry compared to all other regions. This happens because, when a single region is comparatively smaller, an entrepreneur who migrates between any two of the evenly distributed regions (which are larger) will see his utility rise. Thus, if exogenous migration occurs to any such region, it will attract more and more entrepreneurs until it becomes an industrialized core.

A consequence of the stability analysis of partial agglomeration is that the QL model distribution patterns with three regions and more cannot be explained by the 2-region model's pitchfork bifurcation (Pflüger, 2004). Instead, it undergoes a primary transcritical bifurcation at the symmetric dispersion equilibrium and a secondary saddle-node bifurcation occurring at a primary branch along the invariant subspace. The existence of a saddle-node implies that entrepreneurs, who are initially partially agglomerated, will become permanently dispersed across all regions if transport costs increase temporarily. Moreover, it is possible that agglomeration becomes stable before dispersion becomes unstable, depending on the level of worker mobility. Thus, from a smooth path where 
transport costs decrease, once symmetric dispersion looses stability, there are two possibilities: (i) if the worker mobility is high, industry converges immediately to partial agglomeration and then smoothly transits towards a single region agglomeration; (ii) if mobility is low, industry immediately agglomerates in a single region.

We have shown that the average utility of the entrepreneurs declines from agglomeration until dispersion, where their average utility is at its lowest. The converse happens to the welfare of farmers. Their average utility is minimal at agglomeration and is highest at dispersion. This evidences a clear trade-off in spatial distributions between entrepreneurs and farmers in terms of social desirability. When we look at the society as a whole, the model exhibits a tendency towards over-agglomeration when transportation costs lie at intermediate levels. The social desirability of more agglomerated distributions is higher when the proportion of farmers is lower, and can be improved for all workers by decreasing the cost of living through lower transportation costs.

\section{Appendix A}

This appendix contains the formal proofs pertaining to Section 2 of the paper.

Proof of Proposition 1 The average nominal wage is the weighted sum of nominal wages in each region, given by (12):

$$
\begin{aligned}
\bar{w} & =\sum_{i=1}^{n} h_{i} w_{i}=\frac{\mu}{\sigma} \sum_{i=1}^{n} h_{i} \sum_{j=1}^{n} \frac{\phi_{i j}\left(\lambda / n+h_{j}\right)}{\phi+(1-\phi) h_{j}}=\frac{\mu}{\sigma} \sum_{j=1}^{n} \sum_{i=1}^{n} h_{i} \frac{\phi_{i j}\left(\lambda / n+h_{j}\right)}{\phi+(1-\phi) h_{j}} \\
& =\frac{\mu}{\sigma} \sum_{j=1}^{n} \frac{\lambda / n+h_{j}}{\phi+(1-\phi) h_{j}} \sum_{i=1}^{n} h_{i} \phi_{i j}=\frac{\mu}{\sigma} \sum_{j=1}^{n} \frac{\lambda / n+h_{j}}{\phi+(1-\phi) h_{j}}\left[\phi+(1-\phi) h_{j}\right]
\end{aligned}
$$

and the result $\bar{w}=\frac{\mu}{\sigma}(1+\lambda)$ follows.

\section{Appendix B}

This appendix contains all the proofs concerning both existence and local stability of equilibria (Section 3).

Proof of Proposition 2 Let $i$ be the core region. We show that $V_{j}<V_{i} \forall j \neq i$. We have, from (16), that:

$$
V_{i}=\frac{\mu}{\sigma}(1+\lambda)+\eta \text { and } V_{j}=\frac{\mu}{\sigma n \phi}\left[\lambda+\phi^{2}(\lambda+n)+\lambda(n-2) \phi\right]+\frac{\mu}{\sigma-1} \ln \phi+\eta .
$$


A straightforward simplification of the inequality $V_{j}<V_{i}$ yields the desired result.

Proof of Proposition 3 Local stability of interior equilibria in $\triangle$ is given by the sign of the real part of the eigenvalues of the Jacobian matrix of the system in (17) at $\left(h_{1}, h_{2}, \ldots, h_{n-1}\right)=\left(\frac{1}{n}, \frac{1}{n}, \ldots, \frac{1}{n}\right)$. At symmetric dispersion, the average utility $\bar{V}$ is invariant in the permutation of any two coordinates, due to symmetry. If we interchange the distributions between region 1 and region $n$ we then have $\bar{V}\left(\frac{1}{n}+\varepsilon, \frac{1}{n}, \ldots, \frac{1}{n}\right)=$ $\bar{V}\left(\frac{1}{n}-\varepsilon, \frac{1}{n}, \ldots, \frac{1}{n}\right)$. But this implies that $\partial_{h_{i}} \bar{V}\left(\frac{1}{n}, \frac{1}{n}, \ldots, \frac{1}{n}\right)=0 .{ }^{25}$ The argument of invariance extends to the indirect utility $V_{i}$ in the permutation of any two coordinates $j \neq i$, which implies that $\partial_{h_{j}} V_{i}\left(\frac{1}{n}, \frac{1}{n}, \ldots, \frac{1}{n}\right)=0, \forall j \neq i$. Finally, symmetry among regions establishes that we must have $\partial_{h_{i}} V_{i}\left(\frac{1}{n}, \frac{1}{n}, \ldots, \frac{1}{n}\right)=\partial_{h_{j}} V_{j}\left(\frac{1}{n}, \frac{1}{n}, \ldots, \frac{1}{n}\right)$. The Jacobian matrix of (17) at the symmetric equilibrium is thus given by:

$$
J=\left[\begin{array}{cccc}
\frac{\partial V_{i}}{\partial h_{i}} & 0 & \ldots & 0 \\
0 & \frac{\partial V_{i}}{\partial h_{i}} & \cdots & 0 \\
\vdots & \vdots & \ddots & \vdots \\
0 & 0 & \ldots & \frac{\partial V_{i}}{\partial h_{i}}
\end{array}\right]
$$

which has a repeated real eigenvalue with multiplicity $n-1$ given by $\partial_{h_{i}} V_{i}\left(\frac{1}{n}, \frac{1}{n}, \ldots, \frac{1}{n}\right)$, and total dispersion is stable if $\partial_{h_{i}} V_{i}\left(\frac{1}{n}, \ldots, \frac{1}{n}\right)<0$.

Replacing $h_{n}=1-\sum_{j=1}^{n-1} h_{j}$ in expression (16) and computing the partial derivative with respect to $h_{i}, \partial_{h_{i}} V_{i}\left(\frac{1}{n}, \ldots, \frac{1}{n}\right)$, we find that total dispersion is stable if:

$$
\begin{array}{r}
\partial_{h_{i}} V_{i}\left(\frac{1}{n}, \ldots, \frac{1}{n}\right) \equiv \frac{\mu n(1-\phi)[(2 n-1) \sigma \phi-\lambda(\sigma-1)(1-\phi)-n \phi+\sigma]}{(\sigma-1) \sigma[(n-1) \phi+1]^{2}}<0 \\
\Leftrightarrow(2 n-1) \sigma \phi-n \phi+\sigma-\lambda(\sigma-1)(1-\phi)<0 .
\end{array}
$$

Solving for $\phi$ finishes the proof.

Proof of Theorem 1 Two conditions are necessary for the study of stability of boundary dispersion. The first condition ensures that empty regions remain empty and, analogously to the proof of Proposition 2, demands that $\left.V_{i}\right|_{B D}-\left.V_{j}\right|_{B D}<0$, where $h_{i}=0$ and $h_{j}=1 / k$. The second condition guarantees that along the boundary $\left(h_{i}=0 ; i=\right.$

\footnotetext{
${ }^{25}$ In this formulation, partial derivatives imply that changes in $h_{i}$ are reflected symmetrically in $h_{n}$.
} 
$1, \ldots, n-k)$ the configuration is stable. Similarly to the proof of Proposition 3 , this condition requires that $\left.\partial_{h_{j}} V_{j}\right|_{B D}<0$.

These two conditions yield, respectively:

$$
\begin{array}{r}
n \sigma \phi[(k-1) \phi+1] \ln \left[\frac{k \phi}{(k-1) \phi+1}\right]+(\sigma-1)(1-\phi)[\lambda(1-\phi)-n \phi]<0, \\
\quad k[n(2 \sigma-1) \phi-\lambda(\sigma-1)(1-\phi)]+n \sigma(1-\phi)<0 .
\end{array}
$$

Solving for $\lambda$, we obtain:

$$
\frac{n[k(2 \sigma-1) \phi+\sigma(1-\phi)]}{k(\sigma-1)(1-\phi)}<\lambda<\frac{n \phi\left\{\sigma[(k-1) \phi+1] \ln \left[\frac{(k-1) \phi+1}{k \phi}\right]+(\sigma-1)(1-\phi)\right\}}{(\sigma-1)(1-\phi)^{2}} .
$$

The strict inequalities are incompatible, and thus boundary dispersion is unstable, if:

$$
\frac{n \phi\left\{\sigma[(k-1) \phi+1] \ln \left[\frac{(k-1) \phi+1}{k \phi}\right]+(\sigma-1)(1-\phi)\right\}}{(\sigma-1)(1-\phi)^{2}}-\frac{n[k(2 \sigma-1) \phi+\sigma(1-\phi)]}{k(\sigma-1)(1-\phi)}<0 .
$$

Eliminating common factors of positive sign and rearranging terms to our convenience, the above inequality becomes:

$$
\begin{array}{r}
{[(k-1) \phi+1]\left\{k \phi \ln \left[\frac{(k-1) \phi+1}{k \phi}\right]-(1-\phi)\right\}<0} \\
\Leftrightarrow \frac{1-\phi}{k \phi}-\ln \left(1+\frac{1-\phi}{k \phi}\right)>0 .
\end{array}
$$

Define $g(x)=x-\ln (1+x)$. It is easy to see that $g(0)=0$ and that $g$ is strictly increasing. Hence, $g(x)>0, \forall x>0$. Noting that $\frac{1-\phi}{k \phi}>0$ finishes the proof.

Proof of Proposition 4 Configurations in $\Delta_{i n v}$ satisfy $h_{1} \in[0,1], h_{j}=\frac{1-h_{1}}{n-1}$ for $j \neq 1$. By replacing the values in (17) and solving for an equilibrium we obtain $\lambda=\lambda^{*}\left(h_{1}\right)$, given by (24) and reproduced here for convenience:

$$
\lambda^{*}\left(h_{1}\right) \equiv \frac{n(\sigma-1)(1-\phi) \phi\left(h_{1} n-1\right)-n \sigma\left[h_{1}(1-\phi)+\phi\right]\left[\phi\left(h_{1}+n-2\right)-h_{1}+1\right] \nu}{(\sigma-1)(1-\phi)^{2}\left(h_{1} n-1\right)},
$$

where $\nu=\ln \left\{\frac{\phi\left(h_{1}+n-2\right)-h_{1}+1}{(n-1)\left[h_{1}(1-\phi)+\phi\right]}\right\}$. Let $h_{1} \in(0,1 / n) \cup(1 / n, 1)$ to exclude equilibria other than partial agglomeration. Notice that $\nu>0$ for $h_{1} \in(0,1 / n)$, while $\nu>0$ for $h_{1} \in$ $(1 / n, 1)$. 
(i). Write $\lambda^{*}\left(h_{1}\right)=(\alpha+\beta \nu) / \gamma$, where:

$$
\begin{aligned}
\alpha & =n(\sigma-1)(1-\phi) \phi\left(h_{1} n-1\right) \\
\beta & =-n \sigma\left[h_{1}(1-\phi)+\phi\right]\left[\phi\left(h_{1}+n-2\right)+1-h_{1}\right] \\
\gamma & =(\sigma-1)(1-\phi)^{2}\left(h_{1} n-1\right),
\end{aligned}
$$

and observe that $\alpha / \gamma>0, \beta<0$ and $\nu / \gamma<0$ to see that $\lambda^{*}\left(h_{1}\right)>0$.

(ii). Calculating the first and second derivatives of $\lambda^{*}\left(h_{1}\right)$ we obtain:

$$
\begin{aligned}
\frac{\partial \lambda^{*}\left(h_{1}\right)}{\partial h_{1}} & =\frac{\alpha_{1}+\beta_{1} \nu}{(\sigma-1)(1-\phi)^{2}\left(h_{1} n-1\right)^{2}}, \\
\frac{\partial^{2} \lambda^{*}\left(h_{1}\right)}{\partial h_{1}^{2}} & =\frac{n \sigma[(n-1) \phi+1]^{2}\left(\alpha_{2}+\beta_{2} \nu\right)}{(\sigma-1)(1-\phi)^{2}\left(h_{1} n-1\right)^{3}\left[h_{1}(1-\phi)+\phi\right]\left[\phi\left(h_{1}+n-2\right)-h_{1}+1\right]},
\end{aligned}
$$

where:

$$
\begin{aligned}
\alpha_{1} & =-(1-\phi)\left(1-h_{1} n\right)[(n-1) \phi+1] \\
\beta_{1} & =h_{1}^{2} n(1-\phi)^{2}-2 h_{1}(1-\phi)^{2}+\phi\{n[(n-3) \phi+2]+3 \phi-4\}+1 \\
\alpha_{2} & =(1-\phi)\left(1-h_{1} n\right)\left[(3-2 n) \phi-h_{1}(n-2)(1-\phi)-1\right] \\
\beta_{2} & =-2(n-1)\left[h_{1}(1-\phi)+\phi\right]\left[\phi\left(h_{1}+n-2\right)-h_{1}+1\right] .
\end{aligned}
$$

The sign of the numerator of $\frac{\partial^{2} \lambda^{*}\left(h_{1}\right)}{\partial h_{1}^{2}}$ is the sign of $F\left(h_{1}\right) \equiv \alpha_{2}+\beta_{2} \nu$. Computing $F^{\prime \prime}\left(h_{1}\right)$, we find that $F(1 / n)=0, F^{\prime}(1 / n)=0$, and $F^{\prime \prime}(1 / n)=0$, and that $F\left(h_{1}\right)$ is concave for $h_{1} \in(0,1 / n)$ and convex for $h_{1} \in(1 / n, 1)$. This implies that the numerator of $\frac{\partial^{2} \lambda^{*}\left(h_{1}\right)}{\partial h_{1}^{2}}$ is negative for $h_{1} \in(0,1 / n)$ and positive for $h_{1} \in(1 / n, 1)$.

Since the denominator of of $\frac{\partial^{2} \lambda^{*}\left(h_{1}\right)}{\partial h_{1}^{2}}$ is positive for $h_{1} \in(0,1 / n)$ and negative for $h_{1} \in(1 / n, 1)$, we conclude that $\partial_{h}^{2} \lambda^{*}\left(h_{1}\right)<0$ which means that $\lambda^{*}\left(h_{1}\right)$ is strictly concave. Hence, at most two partial agglomeration equilibria exist.

Calculate the limits of $\lambda^{*}\left(h_{1}\right)$ and its first derivative as $h_{1}$ approaches $1 / n$ :

$$
\begin{aligned}
\lim _{h_{1} \rightarrow \frac{1}{n} \pm} \lambda^{*}\left(h_{1}\right) & =\frac{\sigma(2 n \phi+1)-n \phi}{(\sigma-1)(1-\phi)}>0 . \\
\lim _{h_{1} \rightarrow \frac{1}{n} \pm} \frac{\partial \lambda^{*}\left(h_{1}\right)}{\partial h_{1}} & =\frac{(n-2) n \sigma}{2(n-1)(\sigma-1)}>0 .
\end{aligned}
$$

From the first limit we establish the continuity of $\lambda^{*}\left(h_{1}\right)$. The fact that the second limit is positive, together with the concavity of $\lambda^{*}\left(h_{1}\right)$, guarantees that $\lambda^{*}\left(h_{1}\right)$ is increasing for $h_{1} \in(0,1 / n)$. Therefore, at most one equilibrium exists for $h_{1} \in(0,1 / n)$. If there are 
two equilibria, both may belong to $(1 / n, 1)$.

Proof of Theorem 2 Analogously to the proof of Proposition 3, the symmetry of the problem ensures that the Jacobian matrix at the partial agglomeration equilibrium with coordinates $\left(h_{1}, \frac{1-h_{1}}{1-n}, \ldots, \frac{1-h_{1}}{1-n}\right)$ is of the form:

$$
J=\left[\begin{array}{cccc}
\frac{\partial f_{1}}{\partial h_{1}} & 0 & \ldots & 0 \\
0 & \frac{\partial f_{j}}{\partial h_{j}} & \cdots & 0 \\
\vdots & \vdots & \ddots & \vdots \\
0 & 0 & \cdots & \frac{\partial f_{j}}{\partial h_{j}}
\end{array}\right]
$$

The eigenvalues of $J$ are given by:

$$
\frac{\partial f_{1}}{\partial h_{1}}=h\left(\frac{\partial V_{1}}{\partial h_{1}}-\frac{\partial \bar{V}}{\partial h_{1}}\right), \text { and } \frac{\partial f_{j}}{\partial h_{j}}=\frac{1-h}{1-n} \frac{\partial V_{j}}{\partial h_{j}}
$$

which must both be negative at partial agglomeration for this configuration to be stable. Using (24) to calculate these derivatives, the stability conditions become, respectively:

$$
\begin{cases}\gamma\left(h_{1}\right) \equiv \frac{(1-\phi)\left(1-h_{1} n\right)-(n-1)\left[h_{1}(1-\phi)+\phi\right] \nu}{\left(1-h_{1} n\right)} & <0 \\ \delta\left(h_{1}\right) \equiv \frac{(1-\phi)\left(h_{1} n-1\right)[(n-1) \phi+1]+\Phi \nu}{\left(h_{1} n-1\right)} & <0 .\end{cases}
$$

We finish the proof by showing that:

(i) When $h_{1} \in(0,1 / n)$ we have $\gamma\left(h_{1}\right)>0$, thus partial agglomeration is unstable regardless of the sign of $\delta\left(h_{1}\right)$. To verify this, notice that, for $h_{1} \in(0,1 / n)$, the denominator of $\gamma\left(h_{1}\right)$ is positive so the sign of $\gamma\left(h_{1}\right)$ is that of the numerator. Call $N\left(h_{1}\right)$ this numerator. Direct calculation shows that:

$$
\frac{\partial N\left(h_{1}\right)}{\partial h_{1}}<0 \Leftrightarrow-(1-\phi)\left[\frac{(1-\phi)\left(1-h_{1} n\right)}{\phi\left(h_{1}+n-2\right)+1-h_{1}}+(n-1) \nu\right]<0,
$$

which is always true since $\nu>0$, that is, the numerator of $\gamma\left(h_{1}\right)$ decreases in $h_{1}$. We also have, noticing that $\nu(1 / n)=0, \lim _{h \rightarrow \frac{1}{n}} N\left(h_{1}\right)=0$. Then, $N\left(h_{1}\right)>0$ and $\gamma\left(h_{1}\right)>0$.

(ii) When $h_{1} \in(1 / n, 1)$ we have $\gamma\left(h_{1}\right)<0$ so that only $\delta\left(h_{1}\right)<0$ needs to be verified for stability. To verify this, notice that, for $h_{1} \in(1 / n, 1)$, the denominator of $\gamma\left(h_{1}\right)$ is negative. From proof of Theorem 2, we have $\lim _{h_{1} \rightarrow \frac{1}{n}} N\left(h_{1}\right)=0$. Also, since $\nu<0$ for $h_{1} \in(1 / n, 1)$, we find that $\frac{d N\left(h_{1}\right)}{d h_{1}}>0$. Therefore, the numerator of $\gamma\left(h_{1}\right)$ is positive for 
$h_{1} \in(1 / n, 1)$ and we conclude that $\gamma\left(h_{1}\right)<0$ if $h_{1} \in(1 / n, 1)$.

\section{Appendix C}

This Appendix contains the formal proofs concerning Section 4 . We denote by $f_{i}(h)$ the right-hand side of equation (17).

Proof of Proposition 5 The conditions required for a transcritical bifurcation (Guckenheimer and Holmes, 2002; pp. 149-150) are as follows:

(T1.) For all values of the bifurcation parameter $\phi$, we must have $f_{i}\left(\frac{1}{n}, \ldots, \frac{1}{n} ; \phi\right)=0$.

This condition is satisfied since total dispersion is always an equilibrium.

(T2.) The Jacobian of $f_{i}(h)$ has a zero eigenvalue at total dispersion. This occurs at the break point $\phi_{b}$ given in $(21)$.

(T3.) At total dispersion and at the break point we must have $\frac{\partial^{2} f_{i}}{\partial h_{i}^{2}}\left(\frac{1}{n}, \ldots, \frac{1}{n} ; \phi_{b}\right) \neq 0$.

The second derivative of $f_{i}$ with respect to $h_{i}$ at the symmetric equilibrium is given by:

$\frac{\partial^{2} f_{i}}{\partial h_{i}^{2}}\left(\frac{1}{n}, \ldots, \frac{1}{n}\right)=\frac{\mu(n-2)(1-\phi)\left\{\phi^{2}[n \sigma(2 \lambda+4 n-3)-2 n(\lambda+n)+\sigma]+\sigma \phi[(3-2 \lambda) n-2]+2 \lambda n \phi+\sigma\right\}}{(\sigma-1) \sigma[(n-1) \phi+1]^{3}}$.

At $\phi=\phi_{b}$, we have:

$$
\frac{\partial^{2} f_{i}}{\partial h_{i}^{2}}\left(\frac{1}{n}, \ldots, \frac{1}{n} ; \phi_{b}\right)=\frac{\mu(n-2)(1-2 \sigma)^{2}}{(\lambda+1)^{2}(\sigma-1)^{3}},
$$

which is positive for $n \geq 3$.

(T4.) At total dispersion and at the break point we must have $\frac{\partial^{2} f_{i}}{\partial h_{i} \partial \phi}\left(\frac{1}{n}, \ldots, \frac{1}{n} ; \phi_{b}\right) \neq 0$.

Again, direct computation yields:

$$
\frac{\partial^{2} f_{i}}{\partial h_{i} \partial \phi}\left(\frac{1}{n}, \ldots, \frac{1}{n} ; \phi_{b}\right)=\frac{\mu(2 \sigma-1)[\lambda-\sigma(\lambda+2 n)+n+\sigma]^{2}}{(\lambda+1)^{2} n(\sigma-1)^{3} \sigma}>0 .
$$

Since all conditions are verified, we conclude that the model undergoes a transcritical bifurcation at the break point $\phi_{b}$.

Proof of Proposition 6 A primary branch satisfies $\lambda^{*}\left(h_{1}\right)$ in equation (??). We use the conditions for a saddle-node bifurcation given by Guckenheimer and Holmes (2002, Theorem 3.4.1). Applied to the QL model, they are as follows: 
(SN1.) At partial agglomeration we must have $\frac{d f}{d h}\left(h_{1} ; \lambda^{*}\left(h_{1}\right) ; \phi_{f}\right)=0$.

In this instance, $f\left(h_{i}\right)$ is the RHS of (17) and the proof of Theorem 13 gives:

$$
\begin{aligned}
\frac{d f}{d h_{1}}\left(h_{1} ; \lambda^{*}\left(h_{1}\right) ; \phi_{f}\right) & =0 \Leftrightarrow \\
\delta & =0,
\end{aligned}
$$

where $\delta$ is as in (25). We rewrite $\delta=0$ as:

$$
\frac{\left(1-\phi_{f}\right)\left(1-h_{1} n\right)\left[(n-1) \phi_{f}+1\right]}{\Phi\left(h_{1} ; \phi_{f}\right)}=\nu\left(h_{1} ; \phi_{f}\right),
$$

where

$$
\begin{aligned}
\Phi\left(h_{1}, \phi_{f}\right) & =h_{1}^{2} n\left(1-\phi_{f}\right)^{2}-2 h_{1}\left(1-\phi_{f}\right)^{2}+\phi_{f}\left\{n\left[(n-3) \phi_{f}+2\right]+3 \phi_{f}-4\right\}+1, \\
\nu\left(h_{1}, \phi_{f}\right) & =\ln \left\{\frac{\phi_{f}\left(h_{1}+n-2\right)-h_{1}+1}{(n-1)\left[h_{1}\left(1-\phi_{f}\right)+\phi_{f}\right]}\right\}
\end{aligned}
$$

and $\phi_{f}$ is the level of freeness of trade at which the interior equilibrium changes stability.

(SN2.) At partial agglomeration, $\frac{d^{2} f}{d h^{2}}\left(h_{1} ; \lambda^{*}\left(h_{1}\right) ; \phi_{f}\right) \neq 0$.

From (24) and (32), we have:

$$
\frac{d^{2} f}{d h^{2}}\left(h_{1} ; \lambda^{*}\left(h_{1}\right) ; \phi_{f}\right)=\frac{\left(h_{1}-1\right) h_{1} \mu(1-\phi)^{2}[(n-1) \phi+1]^{2} \Gamma}{(\sigma-1)\left[h_{1}(1-\phi)+\phi\right]^{2}\left[\phi\left(h_{1}+n-2\right)-h_{1}+1\right]^{2} \Phi},
$$

where $\Gamma(h, \phi)=h_{1}^{2}(n-2)(1-\phi)^{2}+2 h_{1}(1-\phi)[(2 n-3) \phi+1]-\phi\{n[(n-5) \phi+2]+5 \phi-4\}-$ 1. The term $\Phi$ is positive. The term $\Gamma\left(h_{1}, \phi\right)$ has only one (meaningful) zero given by:

$$
h_{1}=h_{1}^{*} \equiv-\frac{\phi(2 n(1-\phi)+3 \phi-4)-\sqrt{n-1}(1-\phi)[(n-1) \phi+1]+1}{(n-2)(1-\phi)^{2}},
$$

which is not compatible with (SN1). By replacing $h_{1}=h_{1}^{*}$ in $(25)$ we obtain:

$$
\frac{d f}{d h_{1}}\left(h_{1}^{*} ; \lambda^{*}\left(h_{1}\right) ; \phi_{f}\right)=\delta\left(h_{1}^{*}\right) \equiv-\frac{[(n-1) \phi+1]^{2}}{(n-2)^{2}} \Xi
$$

where:

$$
\Xi=(n-2)[(\sqrt{n-1}+2) n-2]+(n-1)(n+2 \sqrt{n-1}) \ln (n-1) .
$$

Since $\Xi>0$, it follows that $\delta\left(h_{1}^{*}\right)<0$. Thus $\frac{d^{2} f}{d h_{1}^{2}}\left(h_{1} ; \lambda^{*}\left(h_{1}\right) ; \phi_{f}\right) \neq 0$. 
(SN3.) At partial agglomeration, $\frac{d f}{d \phi}\left(h_{1} ; \lambda^{*}\left(h_{1}\right) ; \phi_{f}\right) \neq 0$.

From (24) and (32), we have:

$$
\frac{d f}{d \phi}\left(h_{1} ; \lambda^{*}\left(h_{1}\right) ; \phi_{f}\right)=\frac{\left(1-h_{1}\right) h_{1} \mu\left(h_{1} n-1\right) \times \Theta}{(\sigma-1) \sigma\left[h_{1}(\phi-1)-\phi\right]\left[\phi\left(h_{1}+n-2\right)-h_{1}+1\right] \times \Phi},
$$

where $\Theta=h_{1}^{2} n(1-\phi)^{2}-h_{1}(1-\phi)\{(n-2) \sigma[(n-1) \phi+1]-2 \phi+2\}-\sigma[(n-1) \phi+1](2 n \phi-$ $3 \phi+1)+\phi\{n[(n-3) \phi+2]+3 \phi-4\}+1<0$. Since the term $h_{1} n-1>0$ for $h_{1} \in(1 / n, 1)$, we can conclude that $d f / d \phi>0$ when evaluated at partial agglomeration and at $\phi_{f}$, ensuring that (SN3) is satisfied. This concludes the proof.

Proof of Proposition 7 We know that total dispersion is stable if $\lambda>\lambda_{b}$, whereas agglomeration is stable if $\lambda<\lambda_{s}$. As a result, both equilibria are simultaneously stable if $\left(\lambda_{b}, \lambda_{s}\right)$ is non-empty. Using (20) and (23), simultaneity of stability then requires $\lambda_{s}-\lambda_{b}>0$ :

$$
\begin{array}{r}
\frac{n \phi[(\sigma-1)(1-\phi)-\sigma \ln \phi]}{(\sigma-1)(\phi-1)^{2}}-\frac{\sigma(2 n \phi+1-\phi)-n \phi}{(\sigma-1)(1-\phi)}>0 \Leftrightarrow \\
-\frac{(1-\phi)[(n-1) \phi+1]+n \phi \log (\phi)}{(\sigma-1)(1-\phi)^{2}}>0 \Leftrightarrow \\
-\frac{(1-\phi)^{2}}{\phi(1-\phi+\ln \phi)}<n,
\end{array}
$$

which concludes the proof.

\section{Appendix D}

This Appendix contains the formal proofs concerning Section 5.

Proof of Theorem 3 The weighted average utility of entrepreneurs, obtained from (15), is given by:

$$
\bar{V}=\frac{\mu}{\sigma}(1+\lambda)+\frac{\mu}{\sigma-1} \sum_{i=1}^{n} h_{i} \ln \left[\phi+(1-\phi) h_{i}\right]+\eta .
$$

Define $f\left(h_{i}\right) \equiv h_{i} \ln \left[\phi+(1-\phi) h_{i}\right]$. Observe that $f:[0,1] \rightarrow \mathbb{R}$ is continuous and twice differentiable, and that:

$$
f^{\prime \prime}\left(h_{i}\right)=\frac{2(1-\phi)\left[\phi+(1-\phi) h_{i}\right]-h_{i}(1-\phi)^{2}}{\left[\phi+(1-\phi) h_{i}\right]^{2}}=\frac{h_{i}(1-\phi)^{2}+2 \phi(1-\phi)}{\left[\phi+(1-\phi) h_{i}\right]^{2}}>0
$$


which means that $f$ is strictly convex.

Now define $g\left(h_{1}, \ldots, h_{n-1}\right) \equiv f\left(1-\sum_{i=1}^{n-1} h_{i}\right)$. The function $g: \mathbb{R}^{n-1} \rightarrow \mathbb{R}$ is convex because it is a composition of a convex function with an affine function.

Therefore, $\sum_{i=1}^{n-1} f\left(h_{i}\right)+g(h)$ is convex as it is a sum of convex functions. We conclude that $\bar{V}: \mathbb{R}^{n-1} \rightarrow \mathbb{R}$ is a convex function of $\left(h_{1}, \ldots, h_{n-1}\right)$. Since $\partial_{h_{i}} \bar{V}\left(\frac{1}{n}, \ldots, \frac{1}{n}\right)=0$ (see proof of proposition 3 ) and $\bar{V}$ is convex, it attains a global minimum at dispersion.

Proof of Theorem 4 Let $f\left(h_{i}\right) \equiv \ln \left[\phi+(1-\phi) h_{i}\right]$. Then:

$$
f^{\prime \prime}\left(h_{i}\right)=-\frac{(1-\phi)^{2}}{[h(1-\phi)+\phi]^{2}}<0,
$$

which means that $f\left(h_{i}\right)$ is strictly concave. Therefore, $\sum_{i=1}^{n-1} \ln \left[\phi+(1-\phi) h_{i}\right]$ is a strictly concave function of $\left(h_{1}, \ldots, h_{n-1}\right)$.

Now define $g\left(h_{1}, \ldots, h_{n-1}\right) \equiv f\left(1-\sum_{i=1}^{n-1} h_{i}\right)$. The function $g: \mathbb{R}^{n-1} \rightarrow \mathbb{R}$ is concave because $f$ is a concave monotonic transformation of a concave function of $\left(h_{1}, \ldots, h_{n-1}\right)$. This implies that $\bar{V}^{L}$, given in (28), is strictly concave.

Each price index $P_{i}$ in (13) is invariant to the permutation of any two region's coordinates. Therefore, we can assert that $\partial_{h_{i}} \bar{V}^{L}\left(\frac{1}{n}, \ldots, \frac{1}{n}\right)=0, \forall i \in N .{ }^{26}$ Given strict concavity, $\bar{V}^{L}$ attains a global maximum at $h=(1 / n, \ldots, 1 / n)$, which concludes the proof.

Proof of Proposition 8 Rewrite the social welfare function $\Omega(h)$ in $(30)$ as:

$$
\Omega(h)=\frac{\epsilon}{\lambda+1}+\frac{\mu}{(\lambda+1)(\sigma-1)}\left[g\left(h_{1}\right)+\ldots+g\left(h_{n}\right)\right]
$$

where $g\left(h_{i}\right) \equiv\left(\frac{\lambda}{n}+h_{i}\right) \ln \left[\phi+(1-\phi) h_{i}\right]$.

The optimization plan for $\Omega(h)$ consists on maximizing $\sum_{i} g\left(h_{1}\right)$ subject to $\sum_{j=1}^{n} h_{i}=$ 1. Write the Lagrangian as $\mathcal{L}=g\left(h_{1}\right)+g\left(h_{2}\right)+\ldots+g\left(h_{n}\right)+\gamma\left(1-h_{1}-\ldots-h_{n}\right)$, where $\gamma$ is the Lagrange multiplier. From the first-order conditions:

$$
g^{\prime}\left(h_{1}\right)=g^{\prime}\left(h_{2}\right)=\ldots=\gamma
$$

We must have $g^{\prime}\left(h_{1}\right)=g^{\prime}\left(h_{2}\right)=\ldots=g^{\prime}\left(h_{n}\right)$. Each $g^{\prime}\left(h_{i}\right)$ is given by:

$$
g^{\prime}\left(h_{i}\right)=\frac{(1-\phi)\left(\frac{\lambda}{n}+h_{i}\right)}{h_{i}(1-\phi)+\phi}+\ln \left[h_{i}(1-\phi)+\phi\right] .
$$

\footnotetext{
${ }^{26}$ For a more formal reasoning, see Proof of Proposition 3 in Appendix B.
} 
The second derivative $g^{\prime \prime}\left(h_{i}\right)$ is given by:

$$
g^{\prime \prime}\left(h_{i}\right)=-\frac{(1-\phi)\left[\lambda-h_{i} n(1-\phi)-\phi(\lambda+2 n)\right]}{n\left[h_{i}(1-\phi)+\phi\right]^{2}},
$$

which has either one zero for $h \in[0,1]$ or none. Therefore, $g^{\prime}\left(h_{i}\right)$ has at most one local extreme. This implies that at most two different values of $h_{i} \in[0,1]$ may satisfy $g^{\prime}\left(h_{i}\right)=\gamma$. The consequence of this is that all potential interior maximizers of $\Omega(h)$ are characterized by a vector $h=\left(h_{1}, h_{2}, \ldots, h_{n}\right)$ such that $k$ of its elements correspond to a share of entrepreneurs equal to $h / k$ and the remaining $n-k$ elements have a share equal to $(1-h) /(n-k)$. This concludes the proof.

\section{Proof of Theorem 5}

(i). Define the summation term of $\Omega(h)$ in $(30)$ as $F(h)=f\left(h_{1}\right)+f\left(h_{2}\right)+\ldots+f\left(h_{n-1}\right)+$ $g\left(h_{n}\right)$, where $h_{n}:\left(h_{1}, h_{2}, \ldots, h_{n-1}\right) \mapsto 1-h_{1}-\ldots-h_{n-1}$. The second derivative of each $f\left(h_{i}\right)$ evaluated at $h_{i}=1 / n$ is given by:

$$
\left.\frac{\partial^{2} f\left(h_{i}\right)}{\partial^{2} h_{i}}\right|_{h_{i}=\frac{1}{n}}=\frac{n(1-\phi)[\phi(\lambda+2 n-1)-\lambda+1]}{[(n-1) \phi+1]^{2}},
$$

which is negative if and only if:

$$
\phi<\phi_{w} \equiv \frac{\lambda-1}{\lambda+2 n-1}
$$

Using (21), it is easily verified that $\phi_{b}<\phi_{w}$. If $\phi<\phi_{b}<\phi_{w}$, symmetric dispersion is stable and $f\left(h_{i}\right)$ is concave. Given that $f: \mathbb{R} \mapsto \mathbb{R}$ is strictly concave, replicating the reasoning from the proof of Theorem 3 allows us to conclude that $g\left(h_{n}\right)=f \circ h_{n}: \mathbb{R}^{n-1} \mapsto \mathbb{R}$ is also strictly concave. Therefore, $F(h)$ is strictly concave for $h=(1 / n, \ldots, 1 / n)$ and $\Omega(h)$, a constant term plus $F(h)$, is also strictly concave at symmetric dispersion when the latter is stable. Since $\Omega(h)$ attains a critical value at symmetric dispersion, we conclude that the latter always attains a local maximum when it is stable.

Evaluating welfare at symmetric dispersion gives us:

$$
\Omega\left(\frac{1}{n}, \ldots, \frac{1}{n}\right)=\frac{1}{\lambda+1}\left[\varepsilon+\frac{\mu(\lambda+1)}{(\sigma-1)} \ln \left(\phi+\frac{1-\phi}{n}\right)\right] .
$$

At agglomeration, welfare is given by:

$$
\Omega\left(h_{i}=1\right)=\frac{1}{\lambda+1}\left[\varepsilon+\frac{\mu \lambda(n-1)}{(\sigma-1) n} \ln \phi\right] .
$$


This implies that agglomeration yields a higher welfare than dispersion dispersion if and only if:

$$
\Delta \Omega \equiv \frac{\lambda(n-1)}{n} \ln \phi-(\lambda+1) \ln \left(\phi+\frac{1-\phi}{n}\right)>0 .
$$

The difference $\Delta \Omega$ is concave in $\phi$, has a zero for $\phi \in(0,1)$ and another at $\phi=1$, and is negative at $\phi=\phi_{b}$. Symmetric dispersion is thus strictly better than agglomeration from a social point of view when the former is stable.

(ii). It can be shown that $\Omega^{\prime}(1)$ is concave in $\phi$ with only one root $\phi_{z} \in(0,1)$ and another at $\phi=1$. Moreover, it is negative when evaluated at the sustain point $\phi_{s}$, which implies that $\phi_{s}<\phi_{z}$. Therefore, there exists a $\phi \in\left(\phi_{s}, \phi_{z}\right)$ where agglomeration is stable and $\Omega^{\prime}(1)<0$, meaning that welfare is higher at another less asymmetric distribution. This concludes the proof.

\section{References}

Ago, T., Isono, I., Tabuchi, T. (2006), "Locational disadvantage of the hub", The Annals of Regional Science, 40 (4), 819-848.

Akamatsu, T., Takayama, Y., Ikeda, K. (2012), "Spatial Discounting, Fourier, and Racetrack Economy: A Recipe for the Analysis of Spatial Agglomeration Models", Journal of Economic Dynamics \&6 Control, 36, 1729-1759.

Baldwin, R., Forslid, R., Martin, P., Ottaviano, G., Robert-Nicoud, F. (2004), Economic Geography and Public Policy, Princeton University Press.

Barbero, J., Zofío, J. L. (2012), "The Multiregional Core-periphery Model: The Role of the Spatial Topology", Networks and Spatial Economics, 16 (2), 469-196.

Behrens, K., Gaigne, C., Ottaviano, G. I., Thisse, J.-F. (2006), "Is remoteness a locational disadvantage?", Journal of Economic Geography, 6 (3), 347-368.

Behrens, K., Robert-Nicoud, F. (2011), "Tempora mutantur: in search of a new testament for NEG", Journal of Economic Geography, 11 (2), 215-230.

Behrens, K., Thisse, J.-F. (2007), "Regional economics: A new economic geography perspective", Regional Science and Urban Economics, 37(4), 457-465.

Berliant, M., Kung, F. (2009), "Bifurcations in Regional Migration Dynamics", Regional Science and Urban Economics, 39, 714-720. 
Bosker, M., Brakman, S., Garretsen, H., Schramm, M. (2010), "Adding geography to the new economic geography: bridging the gap between theory and empirics", Journal of Economic Geography, 10 (6), 793-823.

Castro, S.B.S.D., Correia-da-Silva, J., Mossay, P. (2012), "The Core-Periphery Model with Three Regions and More", Papers in Regional Science, 91 (2), 401-418.

Commendatore, P., Kubin, I., Sushko, I. (2015a), "Typical bifurcation scenario in a three region identical new economic geography model", Mathematics and Computers in Simulation, 108, 63-80.

Commendatore, P., Filoso, V., Grafeneder-Weissteiner, T., Kubin, I. (2015), "Towards a multiregional NEG framework: comparing alternative modelling strategies" In Complexity and Geographical Economics (pp. 13-50). Springer International Publishing.

Fabinger, M. (2015), "Cities as solitons: Analytic solutions to models of agglomeration and related numerical approaches", Available at SSRN 2630599.

Forslid, R., Ottaviano, G. (2003), "An Analytically Solvable Core-Periphery Model", Journal of Economic Geography, 3, 229-240.

Forslid, R., Okubo, T. (2012), "On the development strategy of countries of intermediate size - An analysis of heterogeneous firms in a multi-region framework", European Economic Review, 56 (4), 747-756.

Fujita, M., Krugman, P., Venables, A. (1999), The Spatial Economy: Cities, Regions and International Trade, Cambridge, MA: MIT Press.

Fujita, M., Mori, T. (2005), "Frontiers of the new economic geography", Papers in Regional Science, 84 (3), 377-405.

Fujita, M., Thisse, J.-F. (2009), "New economic geography: an appraisal on the occasion of Paul Krugman's 2008 Nobel Prize in Economic Sciences", Regional Science and Urban Economics, 39 (2), 109-119.

Gaspar, J.M., Castro, S.B.S.D., Correia-da-Silva, J. (2013), "The Footloose Entrepreneur Model with 3 regions", FEP Working Papers, 496.

Guckenheimer, J., Holmes, P. (2002), Nonlinear Oscillations, Dynamical Systems, and Bifurcations of Vector Fields, Springer-Verlag.

Ikeda, K., Akamatsu, T., Kono, T. (2012), "Spatial period-doubling agglomeration of a core-periphery model with a system of cities", Journal of Economic Dynamics and Control, 36 (5), 754-778. 
Ikeda, K., Murota, K., Akamatsu, T., Kono, T., Takayama, Y. (2014), "Self-organization of hexagonal agglomeration patterns in new economic geography models", Journal of Economic Behavior \& Organization, 99, 32-52.

Krugman, P. (1991), "Increasing Returns and Economic Geography", Journal of Political Economy, 99 (3), 483-499.

Krugman, P. (1993), "First nature, second nature, and metropolitan location", Journal of Regional Science, 33 (2), 129-144.

Mossay, P. (2013), "A theory of rational spatial agglomerations", Regional Science and Urban Economics, 43 (2), 385-394.

Ottaviano, G., Tabuchi, T., Thisse, J.-F. (2002), "Agglomeration and Trade Revisited", International Economic Review, 43 (2), 40-435.

Oyama, D. (2009), "Agglomeration under forward-looking expectations: Potentials and global stability", Regional Science and Urban Economics, 39 (6), 696-713.

Puga, D. (1999), "The Rise and Fall of Regional Inequalities", European Economic Review, 43, 303-334.

Pflüger, M. (2004), "A simple, analytically solvable, Chamberlinian agglomeration model", Regional Science and Urban Economics, 34, 565-573.

Pflüger, M., Südekum, J. (2008a), "A synthesis of footloose-entrepreneur new economic geography models: when is agglomeration smooth and easily reversible?", Journal of Economic Geography, 8 (1), 39-54.

Pflüger, M., Südekum, J. (2008b), "Integration, agglomeration and welfare", Journal of Urban Economics, 63, 544-566.

Picard, P. M., Tabuchi, T. (2010), "Self-organized agglomerations and transport costs", Economic Theory, 42 (3), 565-589.

Tabuchi, T. (2014), "Historical trends of agglomeration to the capital region and new economic geography", Regional Science and Urban Economics, 44, 50-59.

Tabuchi, T., Thisse, J.-F. (2011), "A new economic geography model of central places", Journal of Urban Economics, 69 (2), 240-252.

Tabuchi, T., Thisse, J.-F., Zeng, D. Z. (2005), "On the number and size of cities", Journal of Economic Geography, 5 (4), 423-448. 